\title{
Origin of Crack Tip Instabilities
}

\author{
M. Marder and Steve Gross \\ Department of Physics and Center for Nonlinear Dynamics \\ The University of Texas, Austin TX 78712
}

\begin{abstract}
This paper demonstrates that rapid fracture of ideal brittle lattices naturally involves phenomena long seen in experiment, but which have been hard to understand from a continuum point of view. These idealized models do not mimic realistic microstructure, but can be solved exactly and understood completely. First it is shown that constant velocity crack solutions do not exist at all for a range of velocities starting at zero and ranging up to about one quarter of the shear wave speed. Next it is shown that above this speed cracks are by and large linearly stable, but that at sufficiently high velocity they become unstable with respect to a nonlinear micro-cracking instability. The way this instability works itself out is related to the scenario known as intermittency, and the basic time scale which governs it is the inverse of the amount of dissipation in the model. Finally, we compare the theoretical framework with some new experiments in Plexiglas, and show that all qualitative features of the theory are mirrored in our experimental results.
\end{abstract}

PACS: 62.20.Mk, 46.30.Nz Submitted to Journal of the Mechanics and Physics of Solids

\section{Introduction}

The classic theory of fracture (FREUND 1990; KANNINEN AND POPELAR 1985) treats cracks as mathematical branch cuts which begin to move when an infinitesimal extension of the crack releases more energy than is needed to create fracture surface. This idea is enormously successful in practice, but conceptually incomplete. We will show that one cannot understand how a crack moves without taking into account the fact that it moves in a fundamentally discrete and not continuous medium. In a lattice there are some velocities for which crack solutions do not exist at all, others where cracks are linearly unstable and accelerate to higher velocities, and others for which crack tips are unstable and break apart altogether. While these conclusions are all compatible with continuum mechanics, they were not predicted by it, and are somewhat surprising.

The experimental observations which motivate this study are 
- that cracks in amorphous brittle materials such as glass or Plexiglas pass almost instantaneously from quasi-static motion to motion at about $10 \%$ of the Rayleigh wave speed (DÖLL AND WEIDMANN 1976; TAKAHASHI, MATSUSHIGE, AND SAKURADA 1984) .

- that they seldom travel faster than $60 \%$ of the Rayleigh wave speed (KOBAYASHI, OHTANI, AND SATO, 1974; RAVI-CHANDAR AND KNAUSS, 1984), although according to continuum theory (FREUND, 1990) this wave speed should be the limiting velocity.

- that at about $40 \%$ of the Rayleigh wave speed, the acceleration of cracks slows sharply, their velocity begins to oscillate (FINEBERG et. al., 1991, 1992; WASHABAUGH AND KNAUSS, 1993), they emit high-frequency acoustic waves (GROSS et. al., 1993), the energy needed to form new crack surface increases dramatically (GREEN AND PRATT, 1974), and the fracture surface shows periodic structure (MECHOLSKY, 1985) correlated with velocity oscillations (FINEBERG, et. al., 1992) . The basic time scale of the velocity oscillations is much larger than the time scale on which the crack snaps atomic bonds, and has been unexplained.

The continuum theory of fracture contains many hints concerning these phenomena, but its predictions have always seemed ambiguous. In the very first detailed calculation concerning dynamic fracture, Yoffe (1951) showed that at around $60 \%$ of the Rayleigh wave speed, a crack should become unstable, since the maximum tensile stress would no longer be directly ahead of the crack, but would instead be off at an angle. The dynamical implications of this calculation were however unclear. Would the crack branch, would it begin oscillatory motion, or do something else? To make matters more puzzling, depending on precisely which component of the stress one monitors, the instability can set in at different velocities, and there is no criterion deciding between the various possibilities (FREUND 1990). For example, Freund (1974) has found that the velocity at which a moving crack consumes enough energy so that two nearly static cracks could travel instead of one fast one is $45 \%$ of the Rayleigh wave speed $c_{R}$, while some recent perturbative calculations (XU AND KEER, 1992; GAO, 1993) find a dynamical instability at around $65 \%$ of $c_{R}$. In addition there are hints of instabilities in calculations coupling thermal and mechanical motion (LANGER, 1993A; LANGER AND NAKANISHI, 1993), and in other simple energy balance arguments (SLEPYAN, 1992; GAO, 1993; SLEPYAN, 1993) .

All of these calculations are constrained by the fact that continuum elasticity is not well suited to describe the microscopic processes by which elastic energy is converted to broken bonds. We believe that understanding how this happens, even in the simplest case, is the key to resolving the qualitative puzzles mentioned above (MARDER AND LIU, 1993; LIU, 1993).

The idealized models of brittle lattice fracture we will solve in this paper are not intended to describe material microstructures realistically, but, since they can be solved analytically, provide solid ground on which to show how the dynamics of fracture alter when discreteness is taken into account. In these models, steady state crack motion is impossible for velocities less than around 30\% of the relevant wave speed, and at around $50 \%$ of this wave speed, steady state crack motion becomes unstable. We will describe the 
precise dynamical way the instability occurs, show some of its consequences after onset, and compare the results with experiment.

The type of instability found in the lattice theory is similar to that known by the term intermittency (MANNEVILLE, 1990), and it occurs in a simple way. The crack tip starts from some initial condition, and tries to settle into a steady state configuration in which it snaps one by one the atomic bonds perpendicular to its direction of motion. Just as it is about to reach this state, a seemingly irrelevant bond parallel to the direction of motion snaps and throws the crack off its course. The crack tip is thrown far away from the steady state configuration, and gathers itself up to try the approach again, repeating the process periodically. One of the surprises in the analysis is that the time scale which governs the approach of the crack tip to the steady state is given by the time for transient perturbations to die out, and is formally just the inverse of the amount of dissipation in the model. Physical systems with small amounts of dissipation should be expected to show oscillations on relatively long time scales. This idea provides a tentative solution to the most perplexing experimental observation.

From a formal point of view the calculations described here build upon the work of Slepyan (1981) and co-workers (KULAMEKHTOVA, SARAIKHIN, AND SLEPYAN, 1984), lattice solutions for dislocations, (ATKINSON AND CABRERA, 1965; CELLI AND FLYTZANIS, 1970; THOMSON, HSIEH, AND RANA, 1971) and upon results of prior numerical simulations (ASHURST AND HOOVER, 1976; SIERADZKI it et. al., 1988; MACHOVÁ, 1992) . An interesting new result is that many of the steady state configurations which have been derived previously are unphysical and do not exist. In addition, the stability of the remaining steady states is examined carefully. The general rule is that any state whose velocity increases when one pulls on it harder is linearly stable; however, there are nonlinear instabilities to watch for as well, and the points where these occur are determined through a combination of analytical and numerical techniques. The smallest negative eigenvalue governing approach of transient configurations to the steady state is calculated and found to be simply the inverse of the coefficient of dissipation in the model.

There are some clear differences between the behavior of the lattice models and experimental systems. The velocities at which various processes occur in the models are all distinctly higher than their supposed counterparts in experiment. Whether these differences can be attributed to the fact that the model is schematic, or whether additional physical processes altogether actually operate in the experiment, will have to be determined in the future. Some of the most important additional processes to consider have to do with the fact that the experiments are conducted in polymeric solids, not simple lattices, and are fully three-dimensional (RICE, BEN-ZION, AND KIM, 1994; PERRIN AND RICE, 1994) while the theory considers only two dimensions. The solutions considered in this paper do not allow dislocations (ZHOU, et. al., 1994) . This fact may curiously make comparison with polymers more appropriate than with crystals, despite the fact that the calculations are carried out in lattices.

Because analytical calculations of crack motion in lattices are elaborate, the paper will proceed in steps. First, in Section 2 we will review the theory of crack motion in a continuum strip. Next, in Section 3 we will present a one-dimensional model which displays almost all the features of the more elaborate cases. In Section 4 we will solve a two-dimensional model in which mass points move with only one degree of freedom (Mode III). In Section 5 the mass points will be allowed to move with two degrees of 


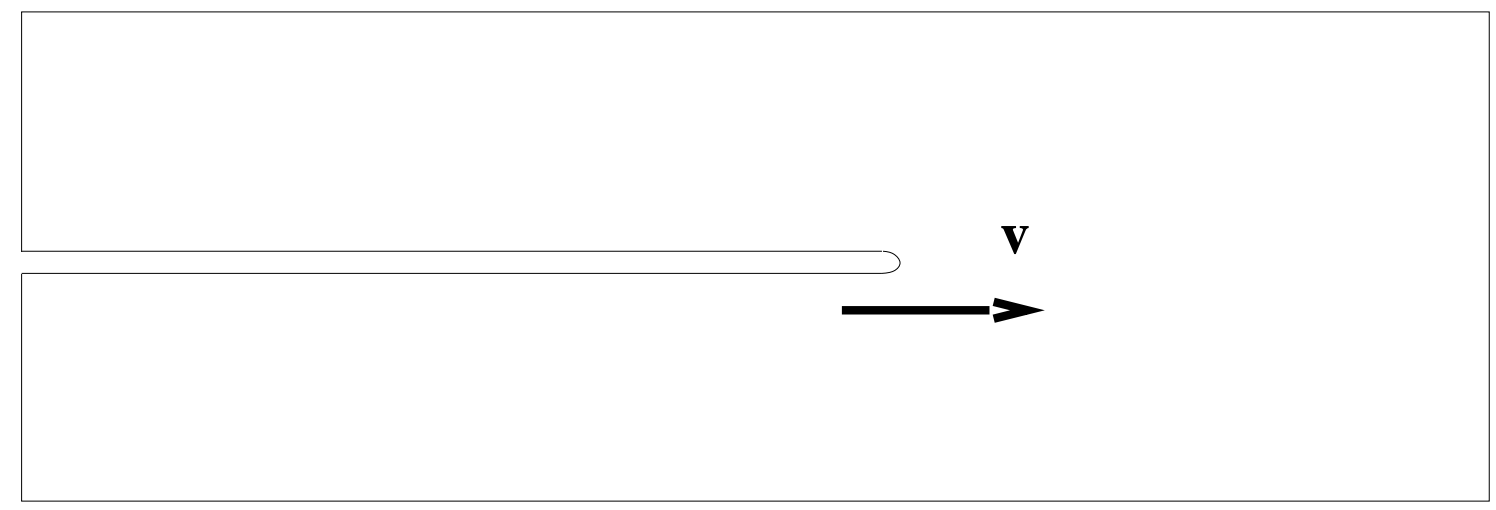

Figure 2.1: All the calculations in this paper will concern a semi-infinite crack moving through the center of an infinite strip.

freedom (Mode I), and the the calculations compared with some new experimental results in Section 6.

\section{Crack motion in a continuum strip}

The successes of dynamic fracture mechanics (FREUND, 1990) provide the backdrop to this paper. The most complete understanding of crack dynamics in a continuum has been obtained for a semi-infinite crack in an infinite plate, with the crack driven by loading on its faces (WILLIS, 1990) . However, we will study a different geometry here, that of a semi-infinite crack in an infinite strip (KNAUSS, 1966; BERGKVIST 1973; BERGKVIST 1974) - There are two reasons for the choice. First, the choice of a strip makes it simpler to compare with numerical simulations over long periods of time, since the effects of top and bottom boundaries are included in the calculation. Second, the strip geometry is the only one in which time-independent loading naturally leads a crack to move in steady state at a constant velocity. In an infinite plate, generic time-independent loading causes a crack to accelerate indefinitely.

What is known about crack motion in a strip (Fig. 2.1)? In certain respects, the geometry is extremely convenient, since when a crack moves some distance $l$ along the middle of a strip, all features of the problem are unchanged except that stressed material in advance of the crack has been converted to unstressed material behind it. For this reason, if elastic energy $W$ is stored per unit length ahead of the crack, then the energy flowing to a steadily moving crack tip to create new surface is also $W$ per unit length. The expected asymptotic solution in a strip is that the crack will move at the velocity $v$ such that

$$
W=\Gamma(v),
$$

where $\Gamma(v)$ is the fracture energy per unit length crack advance. There is an apparent problem with this picture. What happens if $\Gamma(v)$ is constant, but $W$ is slightly more than $\Gamma$ ? In this event, the crack absorbs the excess energy by a continual slow acceleration, 


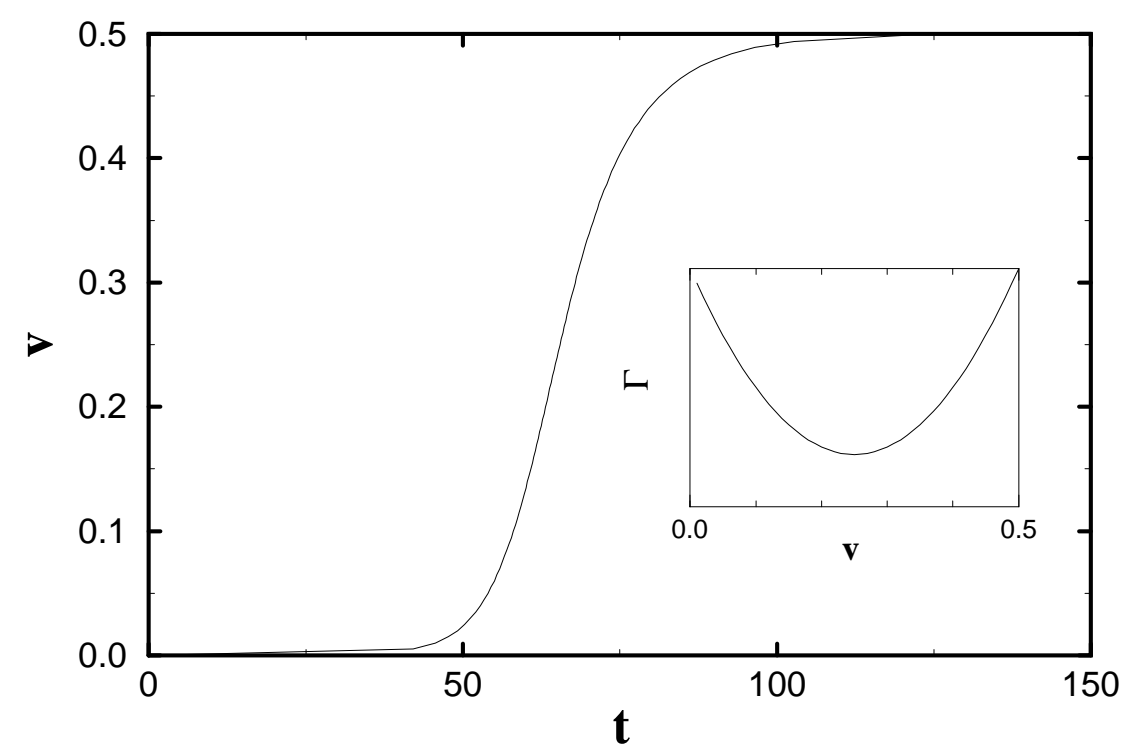

Figure 2.2: Solution of Eq. (2.2) with $\Gamma$ given by Eq. (2.3) and shown in inset, for $W_{0}=1, \rho=1, c_{R}=.9, w=1$, and $v(t=0)=1 \times 10^{-6}$. A long period in which the crack struggles to move is followed by a period of rapid acceleration, and a final approach to steady motion.

moving adiabatically up through steady states and asymptotically approaching a terminal velocity given by a the Rayleigh wave speed (MARDER, 1991; LIU AND MARDER, 1991) . An approximate equation of motion which describes such cases is (LIU AND MARDER, 1991)

$$
\dot{v}=\frac{c_{l}^{2}}{w}[1-\Gamma(v) / W]\left(1-v^{2} / c_{R}^{2}\right)^{2}
$$

with $w$ the half-width of the strip, $c_{R}$ the Rayleigh wave speed, and $c_{l}$ the longitudinal wave speed. The right hand side of Eq. (2.2) is a simple approximation of elaborate analytical expressions; it is only accurate to around $10 \%$, and that only when the dimensionless group $\dot{v} w / c_{l}^{2}$ is small, but is very useful for qualitative analysis.

The particular form of $\Gamma(v)$ is not specified by continuum elasticity, and by varying $\Gamma(v)$ one has the potential to find a wide range of fracture dynamics. Experiments show a very rapid jump in velocity at the onset of fracture, a fact we will explain later as a consequence of the lattice theory, but one can also explain it in the context of the continuum by studying a case in which fracture energy initially decreases with velocity, and then increases again. Taking

$$
\Gamma(v)=\Gamma(0)-W_{0}\left(\frac{v}{2 c_{R}}-\left(\frac{v}{c_{R}}\right)^{2}\right)
$$

leads to the fracture dynamics shown in Fig. 2.2. States at $v=0$ for $W$ slightly less than $\Gamma(0)$ are actually metastable, since crack motion would be possible if it could be initiated. 


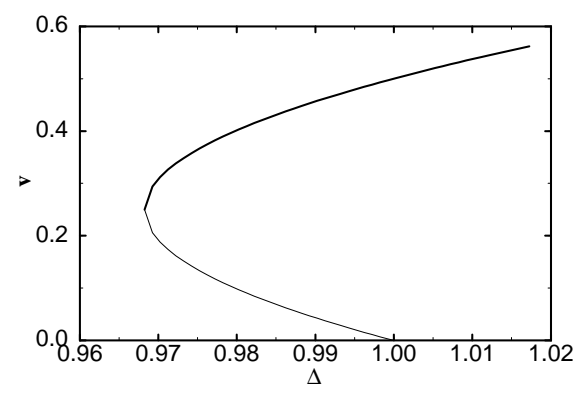

Figure 2.3: Solution of Eq. (2.5) with $\Gamma(0) / W_{0}=1$ and $c_{R}=1$. Only the solutions indicated by the thick line are stable.

Another way to look at the case in which fracture energy $\Gamma$ initially decreases as a function of $v$ is to ask what steady velocities are possible as a function of the energy stored in the strip far ahead of the crack. In order to make a connection with results to be obtained in lattices, define

$$
\Delta=\sqrt{W / \Gamma(0)}
$$

so that $\Delta$ is proportional to the vertical displacement of the sides of the strip ahead of the crack. Then solving Eq. (2.1) and Eq. (2.3) for $v / c_{R}$ gives

$$
\sqrt{\frac{\Gamma(0)}{W_{0}}\left(\Delta^{2}-1\right)+\frac{1}{16}}+\frac{1}{4}=\frac{v}{c_{R}},
$$

which is pictured in Fig. 2.3. The point of this calculation is to show that if fracture energy is initially a decreasing function of velocity, there must be a forbidden band of steady state velocities; cracks which start on the lower branch shown in Fig. 2.3 quickly accelerate to the upper one, meaning that such fractures are activated.

Thus within continuum theory one may have a range of velocities in which cracks are unstable and accelerate rapidly towards some minimum stable velocity. However, lattice theories predict something even more severe: that there is a range of velocities in which steadily moving solutions do not exist at all. 


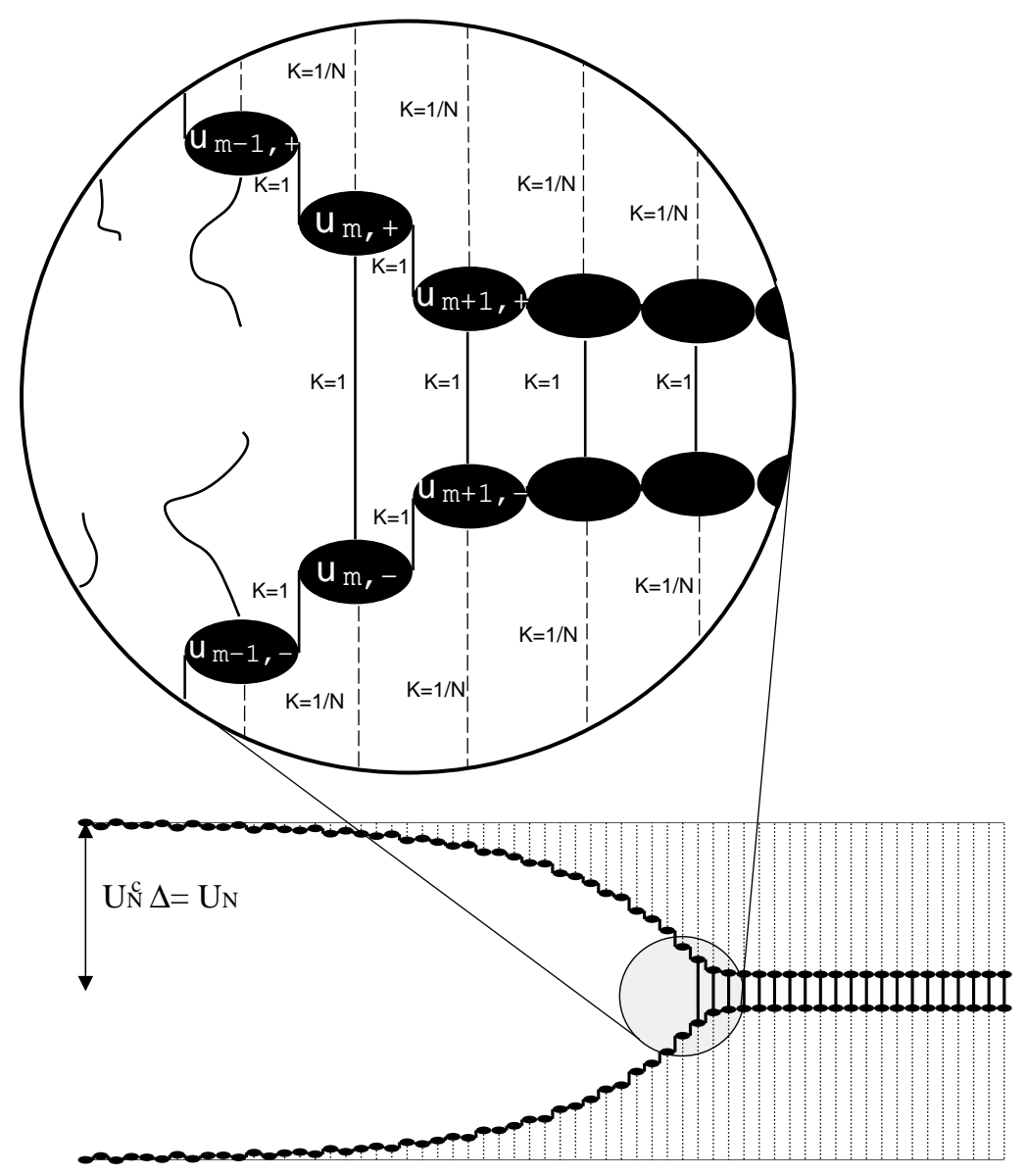

Figure 3.1: This one-dimensional model mimics the motion of a crack in a strip, incorporating effects of discreteness. One can view it as a model for the atoms lying just along the surface of a crack. The mass points are only allowed to move vertically, and are tied to their neighbors with springs which break when they exceed a certain extension. The lower portion of the figure shows an actual solution of the model, using Eq. (3.28), at $v=0.5$, $\mathrm{b}=0.01$.

\section{One-dimensional model}




\subsection{Definition and Energetics}

In order to mimic the motion of a crack in a strip, including the effects of underlying discreteness, but otherwise making the calculation as simple as possible, consider the model shown in Fig. 3.1. One can view it as a model for the atoms lying just along a crack surface. They are tied to nearest neighbors by elastic springs, with spring constant $K=1$, and tied to a line of atoms on the other side of the crack line by similar springs, which however snap when extended past some breaking point. The lines of atoms are being pulled apart by weak springs of spring constant $K=1 / N$. These weak springs are meant schematically to represent $N$ vertical rows of atoms pulling in series, and in later sections will be treated more realistically. The equation which describes the upper row of mass points in this model is

$$
\ddot{u}_{m,+}= \begin{cases}u_{m+1,+}-2 u_{m,+}+u_{m-1,+} & \text { Elastic coupling to neighbors } \\ +\frac{1}{N}\left(U_{N}-u_{m,+}\right) & \text { Driven by displacing edges of strip } \\ +\left(u_{m,-}-u_{m,+}\right) \theta\left(2 u_{f}-\left|u_{m,-}-u_{m,+}\right|\right) & \text { Bonds which snap } \\ -b \dot{u}_{m,+} & \text { Dissipation. }\end{cases}
$$

There are a few terms that need discussion. First, $\theta$ is a step function, and the term containing it describes bonds which snap when their total extension reaches a distance $2 u_{f}$, where $u_{f}$ is a fracture distance. Second, a small amount of dissipation has been added, the term proportional to $b$. Originally this term was included for formal reasons in order to make Fourier transforms well defined, but it will eventually turn out to have physical importance. The amount of dissipation will usually be taken to be vanishingly small. Third, the height that mass points reach after the crack has passed is $U_{N}$, and this term describes the force driving crack motion.

In the discussion that follows, some of the equations will have boxes to the left, like Eq. (3.2). These are equations that are true regardless of the particular lattice that is being considered, and will be taken over without change in later sections.

As $N$ varies, the natural scale on which a displacement $U_{N}$ is able to drive crack motion varies, so one should find the natural dimensionless constant which governs crack motion. The question to ask is, when is enough energy stored in the strip, per lattice spacing far to the right of the crack, to break one bond along the crack line? An important physical quantity to define in answering this question is obtained by going far to the right of the crack, and taking the ratio of the displacement of the atom just above the crack line, $U_{\text {right }}$ to the total displacement at the top of the strip $U_{N}$. Denote this ratio by

$$
Q_{0} \equiv \frac{U_{\text {right }}}{U_{N}}
$$

Suppose that mass points far to the left and far to the right of the crack are stationary, and that dissipation is negligible. Far to the right of the crack, one has that $U_{\text {right }}=u_{m+}=$ $-u_{m-}$, and so balancing forces on masses with $m \gg 0$ gives

$$
\begin{gathered}
2 U_{\text {right }}=\frac{1}{N}\left(U_{N}-U_{\text {right }}\right) \\
\Rightarrow Q_{0}=\frac{1}{2 N+1} .
\end{gathered}
$$


Remembering a factor of two for the upper and lower halves of the strip, the energy per bond for $m \gg 0$ is therefore

$$
\begin{gathered}
2 \frac{1}{2} \frac{1}{N}\left(U_{N}-U_{\text {right }}\right)^{2}+\frac{1}{2}\left(2 U_{\text {right }}\right)^{2} \\
\Rightarrow E_{\text {right }}=2 Q_{0}\left(U_{N}\right)^{2} .
\end{gathered}
$$

Far to the left of the crack, one simply has that

$$
U_{\text {left }}=U_{N} \text {. }
$$

The energy per bond for $m \ll 0$ is just that needed to stretch the spring between $u_{m+}$ and $u_{m}$ - from rest to breaking, and is just

$$
E_{\mathrm{break}}=2 u_{f}^{2}
$$

Assuming there is no other sink of energy, one must have that

$$
2 Q_{0}\left(U_{N}\right)^{2}=2 u_{f}^{2} .
$$

Therefore, the minimum value of $U_{N}$ at which enough energy is stored to the right of the crack so as to be able to snap the bonds along the crack line is

$$
U_{N}^{c}=\frac{u_{f}}{\sqrt{Q_{0}}}
$$

For this reason, it is convenient to define a dimensionless measure of how far one has pulled the edges of the strip,

$$
\Delta \equiv \frac{U_{N} \sqrt{Q_{0}}}{u_{f}} .
$$

By definition, energy balance requires that steady crack motion is only possible for $\Delta \geq 1$, and a system in which a crack moved when $\Delta=1$ would have to be perfectly efficient in turning potential energy into crack motion.

\subsection{Wiener-Hopf solution for steady states}

While the preceding calculations motivate the definition Eq. (3.10), they are based upon false assumptions. When a crack moves in steady state, Slepyan (1981) first showed that the mass points far from its tip are necessarily in motion. As a result the energy accounting carried out above is wrong, and crack motion is only possible for $\Delta>1$. The goal now is to examine steady states in detail.

A steady state in a lattice is more complicated than one in a continuum; it is a configuration which repeats itself after a time interval $1 / v$, but moved over by one lattice spacing. In steady state, one has the symmetries

$$
u_{m+}=-u_{m-},
$$




$$
u_{m+}(t)=u_{0+}(t-m / v),
$$

which means that all spatial behavior is contained in the time history of any single mass point. Take this mass point to be $u_{0+}$, and denote it simply by $u(t)$. Using Eq. (3.13) and Eq. (3.12) in Eq. (3.1) gives

$$
\ddot{u}=u(t-1 / v)-2 u+u(t+1 / v)+\frac{1}{N}\left(U_{N}-u\right)-2 u \theta\left(2 u_{f}-2|u|\right)-b \dot{u} .
$$

Eq. (3.14) can be solved analytically using Wiener-Hopf methods (NOBLE, 1959) . Here is the solution.

There must be some first time at which $u(t)$ rises to 1 and the $\theta$ function vanishes. Let us take this time to be $t=0$. Assuming that $u$ rises above the height of 1 once and remains above it for good, one can write

$$
\ddot{u}=u(t-1 / v)-2 u+u(t+1 / v)+\frac{1}{N}\left(U_{N} e^{-\alpha|t|}-u\right)-2 u \theta(-t)-b \dot{u} .
$$

Eq. (3.15) does not quite follow from Eq. (3.14), since the factor $\exp (-\alpha|t|)$ has appeared. It is introduced just to make Fourier integrals converge, and $\alpha$ will tend to zero at the end of the calculation.

Everything in Eq. (3.15) can be Fourier transformed in a straightforward way except for the term $\theta(t) u(t)$. Simply define

$$
U^{-}(\omega)=\int d t e^{i \omega t} \theta(-t) u(t)
$$

and

$$
U^{+}(\omega)=\int d t e^{i \omega t} \theta(t) u(t)
$$

so that $U(\omega)$, the Fourier transform of $u(t)$, is

$$
U(\omega)=U^{+}(\omega)+U^{-}(\omega)
$$

The crucial observation is that $U^{+}$is free of poles in the upper half complex $\omega$ plane, while $U^{-}$is free of poles in the lower half plane, since the integrals Eq. (3.16) are obviously convergent in these cases.

Using these definitions, one can now transform Eq. (3.15) to read

$$
\begin{gathered}
-\omega^{2} U=2(\cos \omega / v-1) U+\frac{U_{N}}{N}\left\{\frac{1}{\alpha+i \omega}+\frac{1}{\alpha-i \omega}\right\}-\frac{U}{N}-2 U^{-}+i \omega b U . \\
\Rightarrow U(\omega) F(\omega)-2 U^{-}(\omega)=-\frac{U_{N}}{N}\left\{\frac{1}{\alpha+i \omega}+\frac{1}{\alpha-i \omega}\right\}
\end{gathered}
$$

with

$$
F(\omega)=\omega^{2}+2(\cos \omega / v-1)-\frac{1}{N}+i \omega b .
$$

Solving for $U^{-}$with the aid of Eq. (3.17) gives

$$
U^{+}(\omega) \frac{F(\omega)}{F(\omega)-2}+U^{-}(\omega)=-\frac{U_{N}}{N[F(\omega)-2]}\left\{\frac{1}{\alpha+i \omega}+\frac{1}{\alpha-i \omega}\right\} .
$$


Define

$$
Q(\omega)=\frac{F(\omega)}{F(\omega)-2}
$$

and use the fact that $\alpha$ is vanishingly small, so one only needs the value of $F(0)=-1 / N$ on the right hand side of Eq. (3.21) to write

$$
Q(\omega) U^{+}(\omega)+U^{-}(\omega)=Q_{0} U_{N}\left\{\frac{1}{\alpha+i \omega}+\frac{1}{\alpha-i \omega}\right\} .
$$

Here $Q_{0}$ is given by Eq. (3.4), and one can easily check that

$$
Q_{0}=Q(0) .
$$

From a formal point of view, Eq. (3.23) is important because all of the lattice models in subsequent sections can be put in exactly this form, with the function $Q$ becoming progressively more complicated as the model becomes more realistic, but everything else remaining precisely the same.

The Wiener-Hopf technique directs one to write

$$
Q(\omega)=\frac{Q^{-}(\omega)}{Q^{+}(\omega)}
$$

where $Q^{-}$is free of poles and zeroes in the lower complex $\omega$ plane and $Q^{+}$is free of poles and zeroes in the upper complex plane. One can carry out this decomposition with the explicit formula

$$
\begin{gathered}
Q^{ \pm}(\omega)=\exp \left[\mp \int d t e^{i \omega t} \theta( \pm t) \int \frac{d \omega^{\prime}}{2 \pi} e^{-i \omega^{\prime} t} \ln Q\left(\omega^{\prime}\right)\right], \\
=\exp \left[\lim _{\epsilon \rightarrow 0} \int \frac{d \omega^{\prime}}{2 \pi} \frac{\ln Q\left(\omega^{\prime}\right)}{i \omega \mp \epsilon-i \omega^{\prime}}\right] .
\end{gathered}
$$

Now separate Eq. (3.23) into two pieces, one of which has poles only in the lower half plane, and one of which has poles only in the upper half plane:

$$
\frac{U^{+}(\omega)}{Q^{+}(\omega)}-\frac{Q_{0} U_{N}}{Q^{-}(0)} \frac{1}{(-i \omega+\alpha)}=\frac{Q_{0} U_{N}}{Q^{-}(0)} \frac{1}{(i \omega+\alpha)}-\frac{U^{-}(\omega)}{Q^{-}(\omega)} .
$$

Because the right and left hand sides of this equation have poles in opposite sections of the complex plane, they must separately equal a constant, C. The constant must vanish, or $U^{-}$and $U^{+}$will behave as a delta function near $t=0$. So

$$
U^{-}(\omega)=U_{N} \frac{Q_{0} Q^{-}(\omega)}{Q^{-}(0)(\alpha+i \omega)}
$$

and

$$
U^{+}(\omega)=U_{N} \frac{Q_{0} Q^{+}(\omega)}{Q^{-}(0)(\alpha-i \omega)}
$$


One now has an explicit solution for $U(\omega)$. Numerical evaluation of Eq. (3.26a), and $U(t)$ from Eq. (3.28) is fairly straightforward, using fast Fourier transforms. However, in carrying out the numerical transforms, it is important to analyze the behavior of the functions for large values of $\omega$. In cases where functions to be transformed decay as $1 / i \omega$, this behavior is best subtracted off before the numerical transform is performed, with the appropriate step function added back analytically afterwards. Conversely, in cases where functions to be transformed have a step function discontinuity, it is best to subtract off the appropriate multiple of $e^{-t} \theta(t)$ before the transform, adding on the appropriate multiple of $1 /(1-i \omega)$ afterwards. A solution of Eq. (3.28) constructed in this manner appears in Fig. 3.1.

\subsection{Relation between $\Delta$ and $v$}

There is an important point which has been forgotten. This solution is only correct if in fact

$$
u(t)=u_{f} \text { at } t=0
$$

because this is supposed to be the moment at which the bond between $u_{0+}$ and $u_{0-}$ breaks. The only parameter in the problem one is free to vary is $U_{N}$, so the condition Eq. (3.29) chooses a value of $U_{N}$, or its dimensionless counterpart, $\Delta$. Once one assumes that the crack moves in steady state at velocity $v$, there is a unique $\Delta$ which makes it possible.

To obtain Eq. (3.29), one needs to require that

$$
\lim _{t \rightarrow 0^{-}} \int \frac{d \omega}{2 \pi} e^{-i \omega t} U^{-}(\omega)=u_{f} .
$$

This integral can be evaluated by inspection. One knows that for positive $t>0$,

$$
\int d \omega \exp [-i \omega t] U^{-}(\omega)=0
$$

, and that any function whose behavior for large $\omega$ is $1 / i \omega$ has a step function discontinuity at the origin. Therefore, Eq. (3.30) and Eq. (3.28a) become

$$
u_{f}=U_{N} Q_{0} \frac{Q^{-}(\infty)}{Q^{-}(0)}
$$

Since from Eq. (3.22) follows that $Q(\infty)=1$, one sees from Eq. (3.26b) that

$$
Q^{-}(\infty)=Q^{+}(\infty)=1
$$

As a result, one has from Eq. (3.32) and the definition of $\Delta$ given in Eq. (3.11) that

$$
\Delta=\frac{Q^{-}(0)}{\sqrt{Q_{0}}}
$$

To make this result more explicit, use Eq. (3.26b) and the fact that $Q(-\omega)=\bar{Q}(\omega)$ to write

$$
Q^{-}(0)=\exp \left[\int \frac{d \omega^{\prime}}{2 \pi} \frac{1}{2}\left[\frac{\ln Q\left(\omega^{\prime}\right)}{\epsilon-i \omega^{\prime}}+\frac{\ln Q\left(-\omega^{\prime}\right)}{\epsilon+i \omega^{\prime}}\right]\right]
$$




$$
\begin{gathered}
=\exp \left[\int \frac{d \omega^{\prime}}{2 \pi}\left[\frac{1}{-2 i \omega^{\prime}} \ln \left\{\frac{Q\left(\omega^{\prime}\right)}{\bar{Q}\left(\omega^{\prime}\right)}\right\}+\frac{\epsilon}{\epsilon^{2}+\omega^{\prime 2}} \ln Q(0)\right]\right] \\
\Rightarrow Q^{-}(0)=\sqrt{Q_{0}} \exp \left[-\int \frac{d \omega^{\prime}}{2 \pi} \frac{1}{2 i \omega^{\prime}} \ln \left\{\frac{Q\left(\omega^{\prime}\right)}{\bar{Q}\left(\omega^{\prime}\right)}\right\}\right] .
\end{gathered}
$$

Placing Eq. (3.37) into Eq. (3.34) gives

$$
\Delta=\exp \left[-\int \frac{d \omega^{\prime}}{2 \pi} \frac{1}{2 i \omega^{\prime}} \ln \left\{\frac{Q\left(\omega^{\prime}\right)}{\bar{Q}\left(\omega^{\prime}\right)}\right\}\right] .
$$

This expression is not completely general because the fracture condition that $U(t=0)$ must equal $u_{f}$ is not completely general. In the lattice considered in Section 5 , one has instead that $U(t=0)$ must equal $2 u_{f} / \sqrt{3}$. Apart from this constant of proportionality everything goes through as above, and one has the general result that

$$
\Delta=C \exp \left[-\int \frac{d \omega^{\prime}}{2 \pi} \frac{1}{2 i \omega^{\prime}}\left\{\ln Q\left(\omega^{\prime}\right)-\overline{\ln Q\left(\omega^{\prime}\right)}\right\}\right],
$$

where $C$ is a constant of order unity that is determined by the geometry of the lattice, equaling 1 for the model of this section and the model of Section 4 , and $2 / \sqrt{3}$ for the model of Section 5. When written in this form, Eq. (3.39) is suitable for numerical evaluation, since there is no uncertainty relating to the phase of the logarithm.

When $b$ becomes sufficiently small, $Q$ is real for real $\omega$ except in the small neighborhood of isolated roots and poles that sit near the real $\omega$ axis. Let $r_{i}^{+}$be the roots of $Q$ with negative imaginary part (since they belong with $\left.Q^{+}\right), r_{i}^{-}$the roots of $Q$ with positive imaginary part, and similarly $p_{i}^{ \pm}$the poles of $Q$. Then one can rewrite Eq. (3.39) as

$$
\Delta=C \sqrt{\prod \frac{r_{i}^{-} p_{i}^{+}}{r_{i}^{+} p_{i}^{-}}}, \text {for } b=0
$$

Together with Eq. (3.28), Eq. (3.39) and Eq. (3.40) constitute the formal solution of the model. Since $Q$ is a function of the steady state velocity $v$, Eq. (3.38) relates the external driving force on the system, $\Delta$, to the velocity of the crack $v$.

It is interesting to plot the function $\Delta(v)$ obtained from Eq. (3.40) (Fig. 3.2). Because all steady states occur for $\Delta>1$, one necessarily concludes that not all energy stored to the right of the crack tip ends up devoted to snapping bonds. The fate of the remaining energy depends upon the amount of dissipation $b$, and the distance from the crack tip one inspects. In the limit of vanishing dissipation $b$, traveling waves leave the crack tip and carry energy off in its wake; the amount of energy they contain becomes independent of $b$. Such a state is depicted in Fig. 3.1, which shows a solution of Eq. (3.28) for $v=0.5$, $N=9$, and $b=0.01$. For all nonzero $b$, these traveling waves will eventually decay, and the extra energy will have been absorbed by dissipation, but the value of $b$ determines whether one views the process as microscopic or macroscopic. 


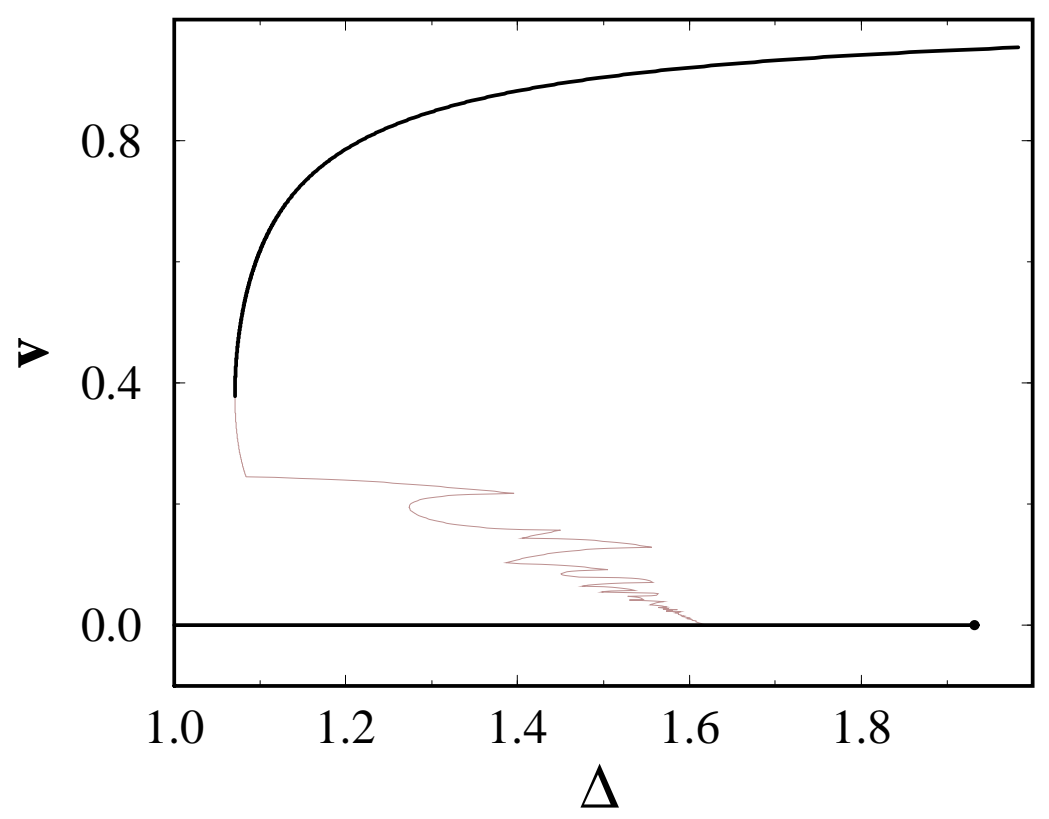

Figure 3.2: The velocity of a crack $v$ is plotted as a function of the driving force $\Delta$ for the one-dimensional model. The calculation is carried out using Eq. (3.40) for $N=100$ and $v=0.5$. The thick upper line indicates physically realizable solutions, and the line along $v=0$ indicates the range of lattice-trapped solutions.

\subsection{Forbidden velocities}

The jagged structure of Fig. 3.2 makes it appear that many different states, emitting different quantities of radiation, can coexist for some values of $\Delta$. This conclusion is largely incorrect, for two reasons. In Appendix III, it is shown that states are linearly unstable whenever $v$ is a decreasing function of $\Delta$. So all the backward-traveling portions of the curve can be ruled out. In addition a final condition has been neglected. Not only must the bond between $u_{0+}$ and $u_{0-}$ reach length $u_{f}$ at $t=0$, but this must be the first time at which that bond stretches to a length greater than $u_{f}$. For $0<v<0.3 \ldots$ (the precise value of the upper limit varies with $b$ and $N$ ) that condition is violated. The states have the unphysical character shown in Fig. 3.3. Masses rise above height $u_{f}$ for $t$ less than 0 , the bond connecting them to the lower line of masses remaining however intact, and then they descend, whereupon the bond snaps. Since the solutions of Eq. (3.15) is unique, but does not in this case solve Eq. (3.14), no solutions of Eq. (3.14) exist at all at these velocities. There is a forbidden band of velocities.

Nevertheless, multiple solutions for some values of $\Delta$ are still possible. The phenomenon of lattice trapping (THOMSON, 1986) allows a crack to sit still in a lattice under some range of external strains, before the first bond holding it snaps and it begins to extend rapidly. The lattice trapped solutions of this model are constructed in Appendix I, 


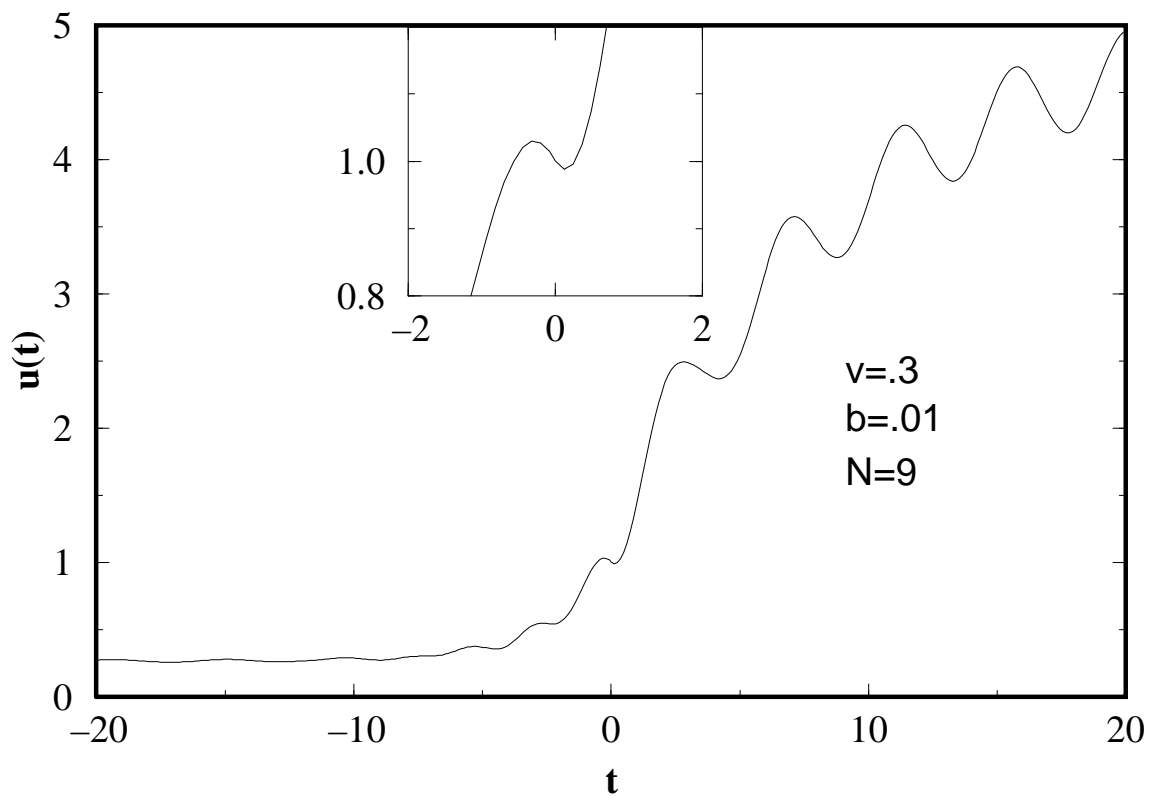

Figure 3.3: The height of $u_{0+}$ is plotted as a function of time for $v=.3$, $b=.01$, and $N=9$. Notice that $u$ rises above $u_{f}=1$ before $t=0$, and is actually descending at the moment when it again passes height $u_{f}$ and is supposed to have broken.

and shown to exist in the range

$$
\frac{\sqrt{3}-1}{\sqrt{2}}=.5176 \ldots<\Delta<\frac{\sqrt{3}+1}{\sqrt{2}}=1.931 \ldots
$$

These bounds do not correspond to the value of $\Delta$ obtained from Eq. (3.40) as $v \rightarrow 0$; that limit is carried out in Appendix II, and shown to be $\Delta=(\sqrt{5}+1) / 2$.

\subsection{Linear Stability}

The stability of steady states can be studied in a straightforward manner, by adding a small term $u^{1}$ to them and linearizing in the perturbation. One finds that even for stable states, transients converge slowly to the final state, at rate $e^{-b t}$, where $b$ is the damping in the model.

This final result can be established in a simple way which relies only upon time reversal symmetries as follows:

Consider any equation of motion for some variables $u_{m}(t)$ of the form

$$
\ddot{u}_{m}(t)=\mathcal{O}_{m}(\vec{u})-b \dot{u}_{m}(t),
$$

where $\mathcal{O}$ is invariant under time translation, and even under time reversal. If one starts with a base solution $u^{0}(t-m / v)$, then perturbations of the form $e^{q t} u_{m}^{1}(t)=e^{q t} u^{1}(t-m / v)$ 
will obey the eigenvalue equation

$$
q^{2} u_{m}^{1}+2 q \dot{u}_{m}^{1}+\ddot{u}_{m}^{1}=\frac{\partial \mathcal{O}_{m}}{\partial \vec{u}} \cdot \vec{u}^{1}-b \dot{u}_{m}^{1}
$$

One easily checks that $\dot{u}^{0}(t-m / v)$ is an eigenfunction with eigenvalue $q=0$. In addition notice that

$$
u_{m}^{1}=\dot{u}^{0}(-t-m / v)
$$

obeys

$$
\begin{gathered}
\ddot{u}_{m}^{1}=\frac{\partial \mathcal{O}_{m}}{\partial \vec{u}} \cdot \vec{u}^{1}+b \dot{u}_{m}^{1} \\
\Rightarrow-2 b \dot{u}_{m}^{1}+\ddot{u}_{m}^{1}=\frac{\partial \mathcal{O}_{m}}{\partial \vec{u}} \cdot \vec{u}^{1}-b \dot{u}_{m}^{1} .
\end{gathered}
$$

Comparing with Eq. (3.43), one sees that to first order in $b$, one has an eigenfunction with eigenvalue $-b$, given simply by time reversing $\dot{u}^{0}$.

This general conclusion is reproduced by the much more detailed analysis of Appendix IV, as discussed in Section 4.3.

\section{Simple Two-dimensional Model (Mode III)}

\subsection{Definition and Energetics of the Model}

The calculation of the previous section will now be extended to a two-dimensional lattice model, depicted in Fig. 4.1. A crack moves in a lattice strip composed of $2(N+1)$ rows of mass points. All of the bonds between lattice points are brittle-elastic, behaving as perfect linear springs until the instant they snap, from which point on they exert no force. The location of each mass point is described by a single spatial coordinate $u(m, n)$, which can be interpreted as the height of mass point $(m, n)$ into or out of the page. The index $m$ takes integer values, while $n$ takes values of the form $1 / 2,3 / 2, \ldots N+1 / 2$. The model is described by the equation

$$
\ddot{u}(m, n)=-b \dot{u}+\frac{1}{2} \sum_{\substack{\text { nearest } \\ \text { neighbors }}} \mathcal{F}\left[u\left(m^{\prime}, n^{\prime}, n^{\prime}\right)-u(m, n)\right],
$$

with

$$
\mathcal{F}(u)=u \theta\left(2 u_{f}-|u|\right)
$$

representing the brittle nature of the springs, $\theta$ the step function, and $b$ the coefficient of a small dissipative term. The boundary condition which drives the motion of the crack is

$$
u(m, \pm[N+1 / 2])= \pm U_{N}
$$




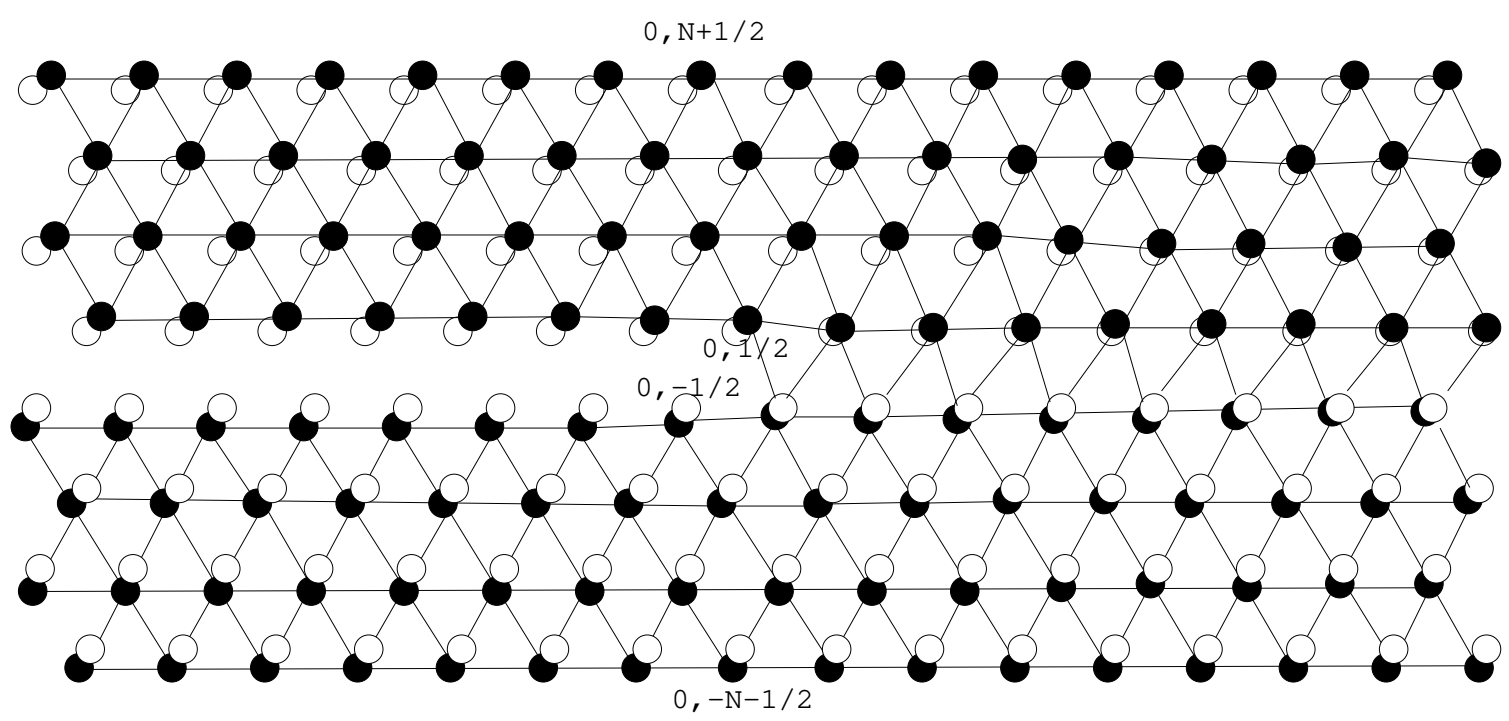

Figure 4.1: Lattice model of fracture. The equilibrium locations of mass points are indicated by the white dots, while the black dots indicate the displacements $u(m, n)$ of mass points out of the page once stress is applied. The top line of dots is displaced out of the page by amount $u(m, N+1 / 2)=\Delta \sqrt{2 N+1}$, and the bottom line into the page by amount $u(m,-N-1 / 2)=-\Delta \sqrt{2 N+1}$. Lines connecting mass points indicate whether the displacement between them has exceeded the critical value of 2 (see Eq. $(4.1 b))$. The crack tip has just reached location $m=0$.

As before, it is important to find the value of $U_{N}$ for which there is just enough energy stored per length to the right of the crack to snap the pair of bonds connected to each lattice site on the crack line. For $m \gg 0$ one has

$$
u(m, n)=\frac{n U_{N}}{N+1 / 2}
$$

so that the energy stored per length in the $2 N+1$ rows of bonds is

$$
\begin{gathered}
\frac{1}{2} \times[2 \text { Upper Bonds } / \text { Site }] \times[\text { Rows }] \times[\text { Spring Constant }] \times U_{\text {right }}^{2} \\
=\frac{1}{2} 2(2 N+1) \frac{1}{2}\left(\frac{U_{N}}{N+1 / 2}\right)^{2} \\
=2 Q_{0}\left(U_{N}\right)^{2},
\end{gathered}
$$

with $Q_{0}=1 /(2 N+1)$ given as before by Eq. (3.4). The energy required to snap two bonds each time the crack advances by unit length is

$$
\frac{1}{2} \frac{1}{2}\left(2 u_{f}\right)^{2}+\frac{1}{2} \frac{1}{2}\left(2 u_{f}\right)^{2}=2 u_{f}^{2}
$$


Therefore, as before, the proper measure of external driving is

$$
\Delta=\frac{U_{N} \sqrt{Q_{0}}}{u_{f}}
$$

a quantity which reaches 1 as soon as there is enough energy to the right of the crack to snap the bonds along the crack line.

\subsection{Reduction to Form of Previous Section}

The techniques used to solve this model were found some time ago by Slepyan (SLEPYAN, 1981). There are differences between details of his solution and ours because Eq. (4.1) describes motion in a strip rather than an infinite plate, and in a triangular rather than a square lattice. The strip is preferable to the infinite plate when it comes time to compare with numerical simulations, while reducing to the simpler infinite plate results in certain natural limits.

Assume that a crack moves in this lattice in steady state, so that one by one, the bonds connecting $u(m, 1 / 2)$ with $u(m+1,-1 / 2)$ or $u(m,-1 / 2)$ break. They break because the distance between these points exceeds the limit set in Eq. (4.1b) and as a consequence of the driving force described by Eq. (4.1c). Supposing that these are the only bonds which snap (an assumption to which we will return later) it is possible to calculate the motion of all the mass points above the line $n=1 / 2$ as a function of the mass points on the line $n=1 / 2$, since in any region where the bonds do not snap the model has simple traveling wave solutions.

In steady state, one has the symmetry

$$
u(m, n, t)=u(m+1, n, t+1 / v)
$$

and also

$$
u(m, n, t)=-u\left(m,-n, t-\left[1 / 2-g_{n}\right] / v\right)
$$

which implies in particular that

$$
u(m, 1 / 2, t)=-u(m,-1 / 2, t-1 / 2 v) .
$$

We have defined

$$
g_{n}= \begin{cases}0 & \text { if } n=1 / 2,5 / 2 \ldots \\ 1 & \text { if } n=3 / 2,7 / 2 \ldots, \\ \bmod (n-1 / 2,2) & \text { in general }\end{cases}
$$

One can now eliminate the variable $m$ entirely from the equation of motion, by defining

$$
u_{n}(t)=u(0, n, t),
$$

and write the equations of motion in steady state as

$$
\begin{aligned}
\ddot{u}_{n}(t)= & \frac{1}{2}\left[\begin{array}{r}
+u_{n+1}\left(t-\left(g_{n+1}-1\right) / v\right)+u_{n+1}\left(t-g_{n+1} / v\right) \\
+u_{n}(t+1 / v)-6 u_{n}(t)+u_{n}(t-1 / v) \\
+u_{n-1}\left(t-\left(g_{n-1}-1\right) / v\right)+u_{n-1}\left(t-g_{n-1} / v\right)
\end{array}\right] \\
& -b \dot{u}_{n}
\end{aligned}
$$


if $n>1 / 2$, and

$$
\begin{aligned}
& \ddot{u}_{1 / 2}(t)= \\
& \frac{1}{2}\left[\begin{array}{c}
+u_{3 / 2}(t)+u_{3 / 2}(t-1 / v) \\
+u_{1 / 2}(t+1 / v)-4 u_{1 / 2}(t)+u_{1 / 2}(t-1 / v) \\
+\left[u_{-1 / 2}(t)-u_{1 / 2}(t)\right] \theta(-t)+\left[u_{-1 / 2}(t-1 / v)-u_{1 / 2}(t)\right] \theta(1 /(2 v)-t)
\end{array}\right] . \\
& -b \dot{u}_{1 / 2}
\end{aligned}
$$

The time at which the bond between $u(0,1 / 2, t)$ and $u(0,-1 / 2, t)$ breaks has been chosen to be $t=0$, so that by symmetry the time the bond between $u(0,1 / 2, t)$ and $u(1,-1 / 2, t)$ breaks is $1 / 2 v$.

For $n>1 / 2$ it is easy to solve the linear set of equations Eq. (4.10a). Fourier transforming in time gives

$$
\begin{aligned}
& \frac{1}{2} u_{n+1}(\omega) {\left[e^{i \omega\left(g_{n+1}-1\right) / v}+e^{i \omega\left(g_{n+1}\right) / v}\right] } \\
&-\omega^{2} u_{n}(\omega)=i b \omega++\frac{1}{2} u_{n}(\omega)\left[e^{i \omega / v}-6+e^{-i \omega / v}\right] \\
& \frac{1}{2} u_{n-1}(\omega)\left[e^{i \omega\left(g_{n-1}-1\right) / v}+e^{i \omega\left(g_{n-1}\right) / v}\right]
\end{aligned} .
$$

Let

$$
u_{n}(\omega)=u_{1 / 2}(\omega) e^{k(n-1 / 2)-i \omega g_{n} /(2 v)} .
$$

Substituting this expression into Eq. (4.11), and noticing that $g_{n}+g_{n+1}=1$ gives

$$
\begin{gathered}
\frac{1}{2} u_{1 / 2}(\omega) e^{k}\left[e^{i \omega\left(g_{n+1}+g_{n}-2\right) /(2 v)}+e^{i \omega\left(g_{n+1}+g_{n}\right) /(2 v)}\right] \\
-\omega^{2} u_{1 / 2}(\omega)=i b \omega u_{1 / 2}(\omega)+\quad \frac{1}{2} u_{1 / 2}(\omega)\left[e^{i \omega / v}-6+e^{-i \omega / v}\right] \\
+\frac{1}{2} u_{1 / 2}(\omega) e^{-k}\left[e^{i \omega\left(g_{n-1}+g_{n}-2\right) /(2 v)}+e^{i \omega\left(g_{n-1}+g_{n}\right) /(2 v)}\right] \\
\Rightarrow \omega^{2}+i b \omega+2 \cosh (k) \cos (\omega /(2 v))+\cos (\omega / v)-3=0 .
\end{gathered}
$$

Defining

$$
z=\frac{3-\cos (\omega / v)-\omega^{2}-i b \omega}{2 \cos (\omega / 2 v)}
$$

one has equivalently that

$$
y=z+\sqrt{z^{2}-1}
$$

with

$$
y=e^{k} \text {. }
$$

One can construct a solution which meets all the boundary conditions by writing

$$
u_{n}(\omega)=u_{1 / 2}(\omega) e^{-i \omega g_{n} / 2 v}\left[\frac{y^{[N+1 / 2-n]}-y^{-[N+1 / 2-n]}}{y^{N}-y^{-N}}\right]+\frac{U_{N}(n-1 / 2)}{N} \frac{2 \alpha}{\alpha^{2}+\omega^{2}} .
$$


This solution equals $u_{1 / 2}$ for $n=1 / 2$, and equals $U_{N} 2 \alpha /\left(\alpha^{2}+\omega^{2}\right)$ for $n=N+1 / 2$, with $\alpha$ sent to zero at the end of the calculation. The most interesting variable is not $u_{1 / 2}$, but the distance between the bonds which will actually snap. For this reason define

$$
U(t)=\frac{u_{1 / 2}(t)-u_{-1 / 2}(t)}{2}=\frac{u_{1 / 2}(t)+u_{1 / 2}(t+1 / 2 v)}{2} .
$$

Rewrite Eq. (4.10b) as

$$
\begin{aligned}
u_{1 / 2}(t)= & \frac{1}{2}\left[\begin{array}{c}
+u_{3 / 2}(t)+u_{3 / 2}(t-1 / v) \\
+u_{1 / 2}(t+1 / v)-4 u_{1 / 2}(t)+u_{1 / 2}(t-1 / v) \\
-2 U(t) \theta(-t)-2 U(t-1 / 2 v) \theta(1 /(2 v)-t)
\end{array}\right] . \\
& -b u_{\dot{1} / 2}
\end{aligned}
$$

Fourier transforming this expression using Eq. (4.18) and defining

$$
U^{ \pm}(\omega)=\int_{-\infty}^{\infty} d \omega e^{i \omega t} U(t) \theta( \pm t)
$$

now gives

$$
u_{1 / 2}(\omega) F(\omega)-\left(1+e^{i \omega / 2 v}\right) U^{-}(\omega)=-\frac{U_{N}}{N} \frac{2 \alpha}{\omega^{2}+\alpha^{2}}
$$

with

$$
F(\omega)=\left\{\frac{y^{[N-1]}-y^{-[N-1]}}{y^{N}-y^{-N}}-2 z\right\} \cos (\omega / 2 v)+1
$$

Next, use Eq. (4.19) in the form

$$
U(\omega)=\frac{\left(1+e^{-i \omega / 2 v}\right)}{2} u_{1 / 2}(\omega)
$$

to obtain

$$
U(\omega) F(\omega)-2\left(\cos ^{2} \omega / 4 v\right) u^{-}(\omega)=-\frac{U_{N}}{N} \frac{2 \alpha}{\omega^{2}+\alpha^{2}}
$$

Writing

$$
U(\omega)=U^{+}(\omega)+U^{-}(\omega)
$$

finally gives

$$
U^{+}(\omega) Q(\omega)+U^{-}(\omega)=U_{N} Q_{0}\left\{\frac{1}{\alpha+i \omega}+\frac{1}{\alpha-i \omega}\right\},
$$

with

$$
Q(\omega)=\frac{F(\omega)}{F(\omega)-1-\cos (\omega / 2 v)} .
$$

To obtain the right hand side of Eq. (4.27) one uses the facts that $F(0)=-1 / N$, and that $\alpha$ is very small, so that the right hand side of Eq. (4.27) is a delta function. 


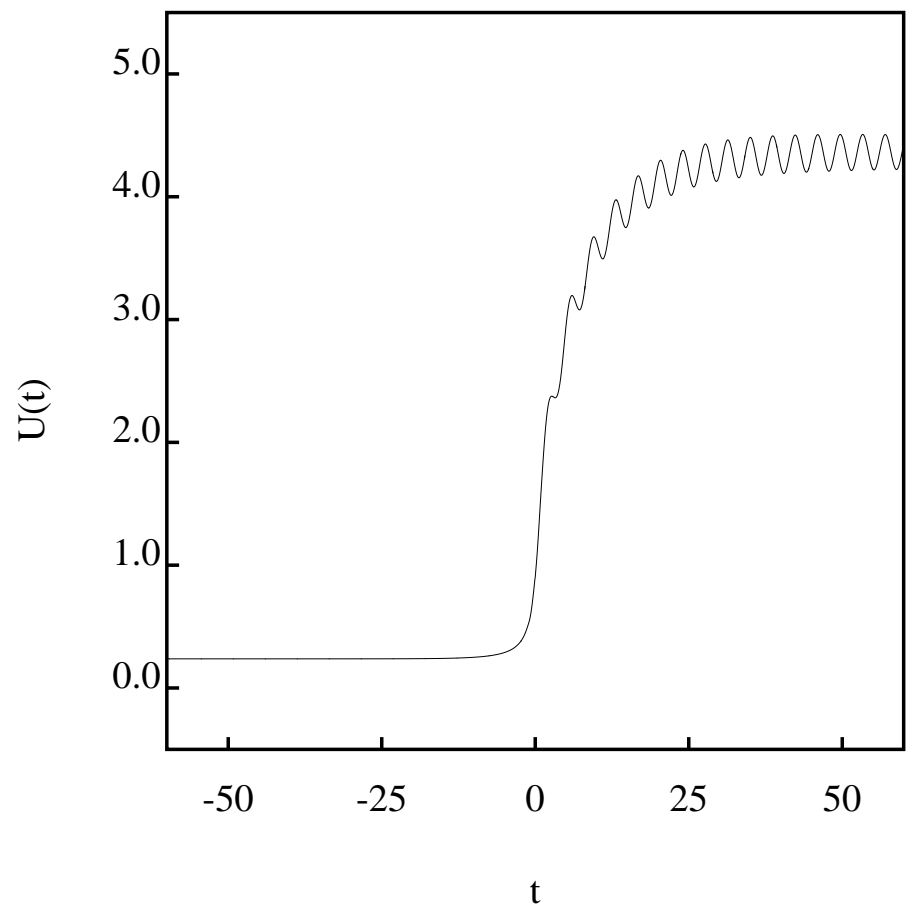

Figure 4.2: Plot of $U(t)$ for $v=.5, N=9$, and $b=0.01$, produced by direct evaluation of Eq. (3.28). Note that mass points are nearly motionless until just before the crack arrives, and that they oscillate afterwards for a time on the order of $1 / b$.

Notice as promised that Eq. (4.27) is identical to Eq. (3.23). Therefore from this point forward the analysis of the Section 3 can be repeated without alteration. In particular Eq. (3.28) gives an explicit expression for $U(\omega)$, and Eq. (3.38) and Eq. (3.40) describe the relation between $\Delta$ and $v$.

Without repeating the calculations, we mention for later reference that a square lattice can be solved in the same manner - it is simpler than the triangular lattice - and the results are the same except that

$$
F(\omega)=\frac{y^{[N-1]}-y^{-[N-1]}}{y^{N}-y^{-N}}-2 z+1
$$

replaces Eq. (4.23) and

$$
Q=\frac{F}{F-2}
$$

replaces Eq. (4.28).

There are three parameters to vary in looking for numerical solutions of Eq. (4.27). The most important is $v$, the velocity of the steady state. In addition, one can also control $N$, the width of the strip, and $b$ the amount of dissipation. There is a natural limit in which many results become independent of $N$ and $b$, the limit of a macroscopic dissipationless 
strip, namely $N \rightarrow \infty$ and $b \rightarrow 0$. No physical results depend upon the order in which these limits are taken, although the integrals one has to perform for Eq. (3.38) look very different. For $1 / b \gg N, \ln Q / \bar{Q}$ is only nonzero near the finite number of points where $Q$ has a pole or a zero. One can use Eq. (3.40) in this case. For $N \gg 1 / b$ these poles and roots merge into branch cuts, and the integrand of Eq. (3.38) becomes smooth. By taking the limit in this way, one can show the equivalence of results in the strip with the results found previously by Slepyan (SLEPYAN, 1981) for a square lattice occupying an infinite plate. In the following discussion, the calculations will be carried out for $N=9$. Although seemingly far from the limit $N \rightarrow \infty$, all physical quantities that have been checked so far change only in the fourth decimal place when $N$ increases from 9 to 100 . So $N=9$ is large enough to give an accurate picture of the behavior of the model, but small enough to make all types of computations rapid.

All the phenomena discussed in relation to the one-dimensional model occur here. A picture of $u(t)$ for $v=.5$ appears in Fig. 4.2, a graph of $\Delta(v)$ appears in Fig. 4.3, and a picture of an unphysical state at $v=.2$ appears in Fig. 4.4. For $N=9$ and $b=0.05$, starting at the wave speed $c=\sqrt{3} / 2$ and working downwards, $\dot{u}(0)$ first becomes negative at $v=0.244$, or $28 \%$ of the wave speed $c$. At lower values of $v$, there is no evidence that the steady states are ever physical. For example, at $v=0.106, \dot{u}(0)$ is positive. However, examining the state, one finds that $u$ earlier rose above $u(0)$, descended below it, and is on the rise again. Stationary states with $v=0$ are physical, and exist up until the point where they are met by the states with $v \neq 0$. Once again, there is a velocity gap, and no steady states exist between $v=0$ and $v=0.244 \ldots$. Above $v=0.577$, or $v / c=.666$, the steady state solutions are unstable, for reasons that will be discussed in a later section.

\subsection{Linear Stability}

The complete calculation of linear stability is carried out for the triangular lattice of this section and the details are relegated to Appendix IV. The results are as follows:

Define

$$
\begin{gathered}
z_{q}=\frac{3-\cos (\omega / v)+(q-i \omega)^{2}-i b \omega}{2 \cos (\omega / 2 v)} \\
y_{q}=z_{q}+\sqrt{z_{q}^{2}-1},
\end{gathered}
$$

and

$$
F_{q}(\omega)=\left\{\frac{y_{q}^{[N-1]}-y_{q}^{-[N-1]}}{y_{q}^{N}-y_{q}^{-N}}-2 z_{q}\right\} \cos (\omega / 2 v)+1 .
$$

Define in addition

$$
U(t)=\frac{u_{1 / 2}^{1}(t)-u_{-1 / 2}^{1}(t+1 / 2 v)}{2}
$$

and restrict attention to modes with the symmetry

$$
u_{1 / 2}^{1}(t)=-u_{-1 / 2}^{1}(t+1 / 2 v),
$$




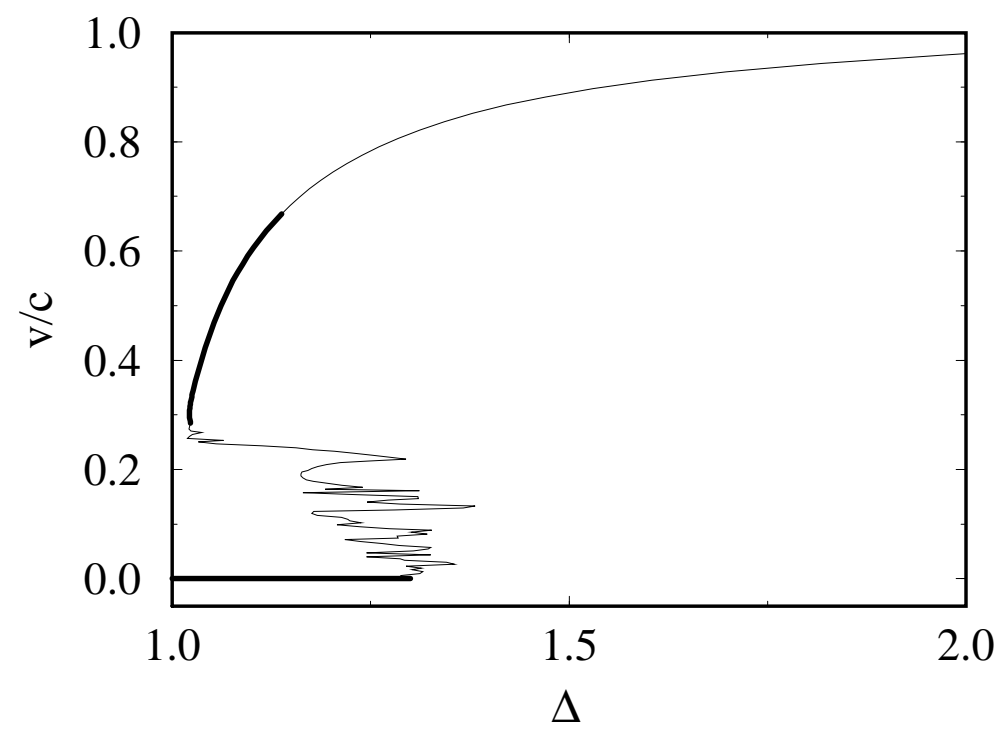

Figure 4.3: Steady state velocities as a function of external strain $\Delta$, calculated for strip half-width $N=9$ and dissipation $b=0$ using Eq. (3.40). The thick lines indicate cases in which the steady states are stable. Zero velocity states at strains $\Delta>1$ correspond to the phenomenon of lattice trapping (THOMSON, 1986).

Then defining

$$
Q_{q}(\omega)=\frac{F_{q}(\omega)}{F_{q}(\omega)-1-\cos (\omega / 2 v)}
$$

one has

$$
U(\omega)=\left(Q_{q}^{+}-Q_{q}^{-}\right) \frac{U_{0}}{\dot{u}^{0}(0)},
$$

with $\dot{u}^{0}(0)$ the velocity of the steady state $u^{0}(t)$ at $t=0$, and $U_{0}$ subject to the consistency condition

$$
U_{0}=\int \frac{d \omega}{2 \pi} U(\omega)
$$

which may be rewritten as

$$
\int \frac{d \omega}{2 \pi}\left(1-Q_{q}^{-}(\omega)\right) \equiv S_{q}=\dot{u}^{0}(0)=S_{0} .
$$

The spectrum of perturbations about steady states is given by the zeroes of $S_{q}-S_{0}$. If $S_{q}-S_{0}$ has a root when the real part of $q$ is positive, the corresponding steady state is unstable.

One consequence of Eq. (4.37) is that any state whose velocity decreases as $\Delta$ increases must be unstable. This result may be established by considering the behavior of $S_{q}$ for small $q$. If the slope of $S_{q}$ is positive at the origin, then there must be a root for positive 


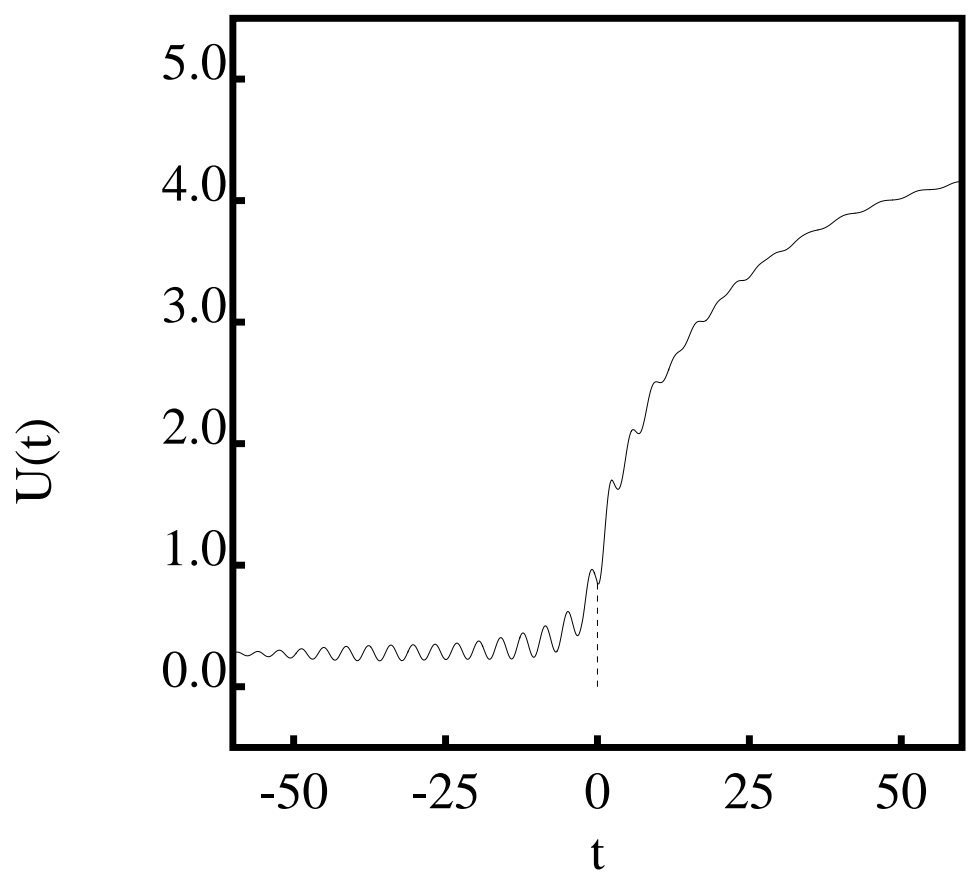

Figure 4.4: Behavior of $u(t)$ for $v=.2, b=0.05$, and $N=9$. Notice that at $t=0$, indicated by the dashed line, $u$ is decreasing, and that it had already reached height 1 earlier. This state is not physical.

$q$, since $S_{0}>0$, and $S$ goes to zero as $q \rightarrow \infty$. The slope $S_{0}^{\prime}$ is given by the following expression, evaluated for the steady state:

$$
S_{0}^{\prime}=-v \frac{\partial}{\partial v} \ln Q_{0}^{-}(0)
$$

or using Eq. (3.28),

$$
S_{0}^{\prime}=-\frac{\partial \ln \Delta}{\partial \ln v}
$$

When accurate numerical evaluation of $S(q)$ is necessary, it can be carried out from the integral

$$
S_{q}=\int \frac{d \omega^{\prime}}{2 \pi} \ln \left[\frac{Q_{0}\left(\omega^{\prime}\right)}{Q_{q}\left(\omega^{\prime}\right)}\right]
$$

The moderately lengthy derivations of Eq. (4.39) and Eq. (4.40) are given in Appendix $\mathrm{V}$.

Fig. 4.5 shows a graph of $S_{q}$. The somewhat surprising triangular shape has a clear physical origin. According to Eq. (4.31a), when $q$ is real and very small, it acts so as to shift the effective value of the damping $b$. Right at $q=-b / 2$, the system is fooled into thinking that $b$ has vanished, and as $q$ decreases further, it is as if $b$ has changed sign, or as if time has started to run backwards. The symmetry of $Q$ implies that

$$
Q(\omega, b)=Q(-\omega,-b)
$$




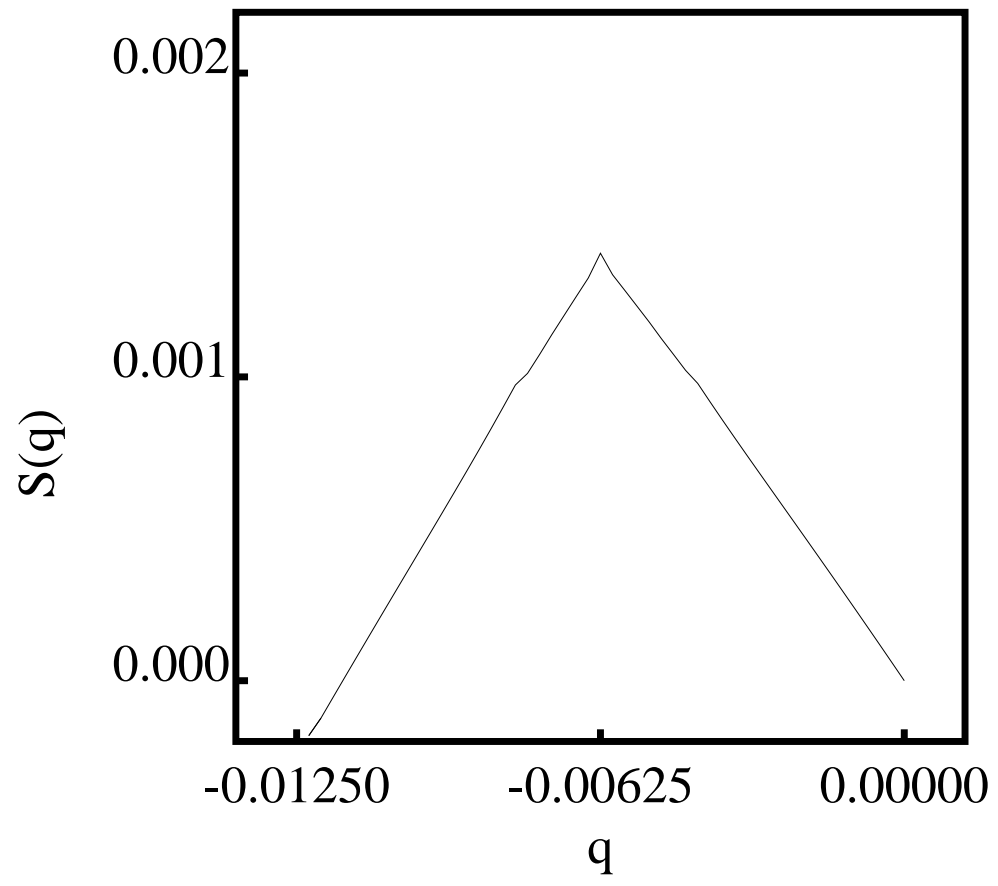

Figure 4.5: The integral on the right side of Eq. (IV.16) is plotted as a function of $q$ for $v=0.5$ and $b=0.0125$.

$$
\Rightarrow Q^{-}(\omega, b) Q^{+}(-\omega,-b)=Q^{-}(-\omega,-b) Q^{+}(\omega, b)
$$

Since the left side of this equation has roots only above the real axis, and the right side only below,

$$
Q^{-}(0,-b)=1 / Q^{+}(0, b)
$$

so using

$$
u^{0}(0)=1=\sqrt{2 N+1} \frac{\Delta}{Q^{+}(0)},
$$

one has that for small positive $\epsilon$

$$
S_{b / 2+\epsilon}^{\prime}=v \frac{\partial}{\partial v} \ln Q_{0}^{+}(0)=\frac{\partial \ln \Delta}{\partial \ln v} .
$$

In short, the sign of the slope of $S_{q}$ changes sign right at $q=-b / 2$, resulting in a stable eigenmode with eigenvalue exactly $-b$. This result is exactly that obtained in Section 3.5. A picture of the mode is given in Fig. 4.6. 


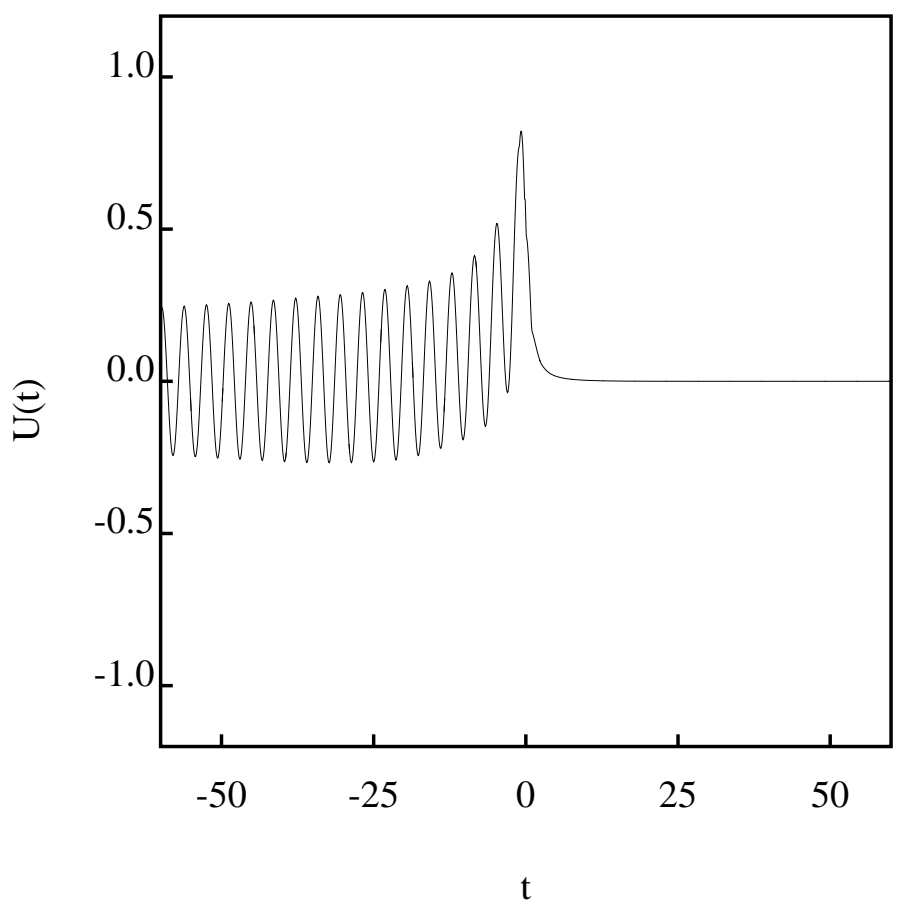

Figure 4.6: The eigenmode $U(t)$ for $q=b$, given by $\dot{u}^{0}(-t)$ is pictured for $v=0.5$ and $b=0.0025$.

\subsection{Nonlinear Instabilities}

Showing that steady states are linearly stable is not sufficient to demonstrate that they are physical. It was assumed in their derivation that the only bonds which break are those which lie on the crack path. From the numerical solutions of Eq. (3.28), one can test this assumption; it fails above a critical velocity. Recall that the sound speed $c$ equals $\sqrt{3} / 2$. For $N=9$, at a velocity of $v_{c} / c=.666 \ldots, \Delta_{c}=1.158 \ldots$, the bond between $u(0,1 / 2)$ and $u(1,1 / 2)$ reaches a distance of $2 u_{f}$ some short time after the bond between $u(0,1 / 2)$ and $u(0,-1 / 2)$ snaps. The steady state solutions strained with larger values of $\Delta$ are inconsistent; only dynamical solutions more complicated than steady states, involving the breaking of bonds off the crack path, are possible. To investigate these states, one must return to Eq. (4.1) and numerically solve the model directly. These simulations have been carried out by Liu (MARDER AND LIU, 1993; LIU, 1993), and some results are contained in Fig. 4.7.

The diagram shows patterns of broken bonds left behind the crack tip. Just above the threshold at which horizontal bonds begin to break, one expects the distance between these extra broken bonds to diverge. The reason is that breaking a horizontal bond takes energy from the crack and slows it below the critical value. The crack then tries once more to reach the steady state, and only in the last stages of the approach does another horizontal bond snap, beginning the process again. This scenario for instability is similar to that known as intermittency in the general framework of nonlinear dynamics (MANNEVILLE, 
$\Delta=1.147$
$\mathrm{v} / \mathrm{c}=0.645$

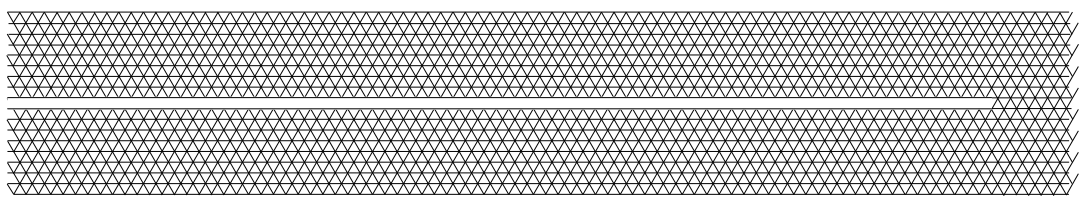

$\Delta=1.165$

$\mathrm{v} / \mathrm{c}=0.630$

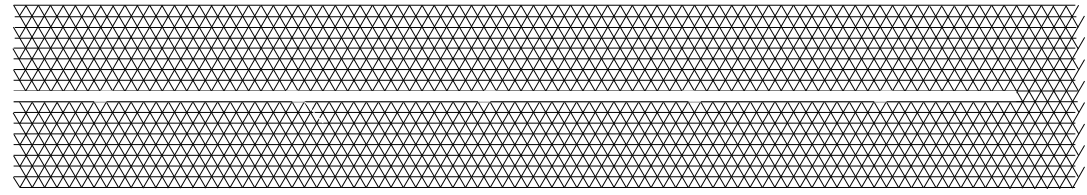

$\Delta=1.376$

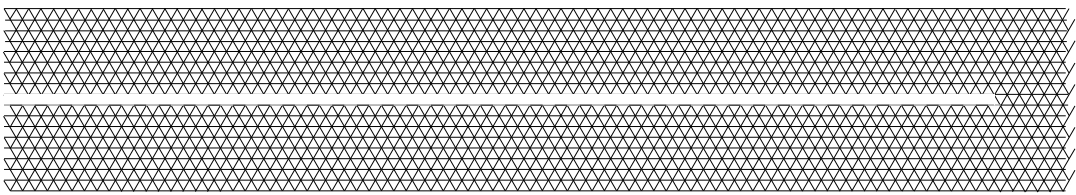

$\Delta=1.835$

$\mathrm{v} / \mathrm{c}=0.775$

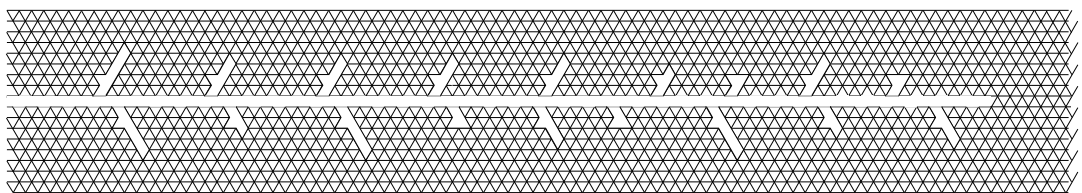

Figure 4.7: Pictures of broken bonds left behind the crack tip at four different values of $\Delta$, from simulations of Liu (.) The top figure shows the simple pattern of bonds broken by a steady-state crack. At a value of $\Delta$ slightly above the critical one where horizontal bonds occasionally snap, the pattern is periodic. All velocities are measured relative to the sound speed $c=\sqrt{3} / 2$. Notice that the average velocity can decrease relative to the steady state, although the external strain has increased. As the strain $\Delta$ increases further, other periodic states can be found, and finally states with complicated spatial structure. The simulations are carried out in a strip with half-width $N=9$, of length 200 and $b=0.01$. The front and back ends of the strip have short energy-absorbing regions to damp traveling waves.

1990) ; the system spends most of its time trying to reach a fixed point which the motion of a control parameter has caused to disappear.

Here is a rough estimate of the distance between broken horizontal bonds. Let $u_{h}(t)$ be the length of an endangered horizontal bond as a function of time. Actually, one needs to view matters in a reference frame moving with the crack tip, so every time interval $1 / v$, one shifts attention to a bond one lattice spacing to the right. When $\Delta$ is only slightly greater than $\Delta_{c}$, the length of such a bond viewed in a moving frame should behave before it snaps, as

$$
u_{h} \sim 2 u_{c}+\frac{\partial u_{h}}{\partial \Delta}\left(\Delta-\Delta_{c}\right)-\delta u e^{-b t} .
$$

Here $\partial u_{h} / \partial \Delta$ means that one should calculate the rate at which the steady-state length of $u_{h}$ would change with $\Delta$ if this bond were not allowed to snap, and $\delta u$ describes how much smaller than its steady-state value the bond ends up after the snapping event occurs. 


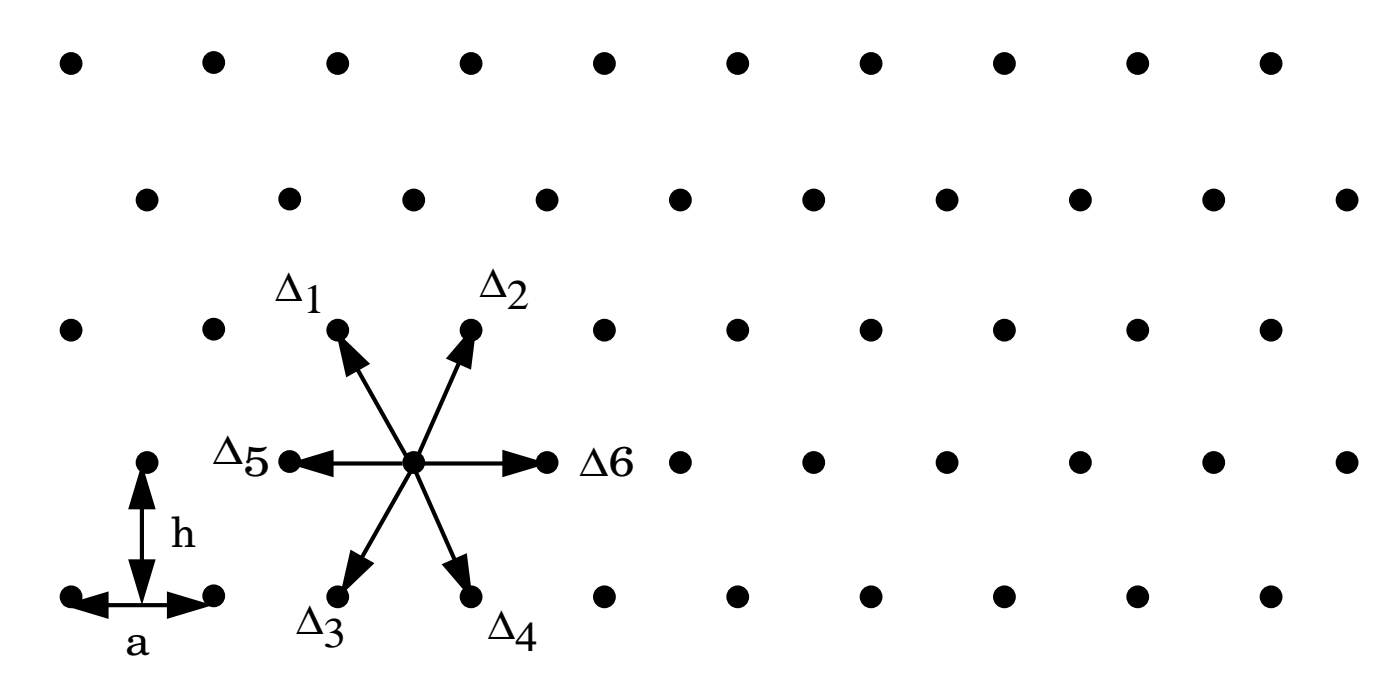

Figure 5.1: Diagram of lattice used in this section

From this expression, one can estimate the time between snapping events by setting $u_{h}$ to $2 u_{f}$ and solving Eq. (4.46) for $t$. The result is that the frequency $\nu$ with which horizontal bonds snap should scale above the critical strain $\Delta_{c}$ as

$$
\nu \sim-b / \ln \left(\frac{1}{\delta u} \frac{\partial u_{h}}{\partial \Delta}\left[\Delta-\Delta_{c}\right]\right),
$$

a result that is consistent with the numerics, but hard to check conclusively. One can calculate numerically that $\partial u_{h} / \partial \Delta=5.5$ for the conditions of Fig. 4.7, but $\delta u$ is hard to find independently. Assuming that Eq. (4.47) is correct, one finds from the second picture of Fig. 4.7 that $\delta u=0.04$. Further increasing the external strain $\Delta$ makes a wide variety of complicated behavior possible, including dendritic patterns, in the lowest panel of Fig. 4.7 that we will see are reminiscent of experiment.

\section{Solution of Fully Two-Dimensional Model (Mode I)}

The triangular lattice solved in Section 4 contains all the physical phenomena we now know to be associated with lattice fracture. Still, it is not very realistic because of the restriction that mass points move in only one dimension. That restriction is relaxed in this section. Unfortunately, the calculations rapidly become so lengthy that they are difficult to explain. For this reason, most of the details are contained in a MAXIMA script which was used to produce the results. However, one can at least study the question of energy balance without too much trouble.

The lattice studied in this section is depicted in Fig. 5.1. Each point has two degrees of freedom associated with motion in the plane. In the absence of external forces, the mass points all sit on a triangular lattice, and they interact linearly with their nearest neighbors. The interaction between two neighbors is a general linear function of both the 
parallel distance between them, and the perpendicular distance between them. The two spring constants $k_{\perp}$ and $k_{\|}$are introduced so as to accommodate a general Poisson ratio. Choosing an arbitrary $k_{\|}$and $k_{\perp}$ results in long-wavelength transverse and longitudinal sound speeds

$$
\begin{aligned}
c_{l}^{2} & =\frac{3 a^{2}}{8 m}\left(k_{\perp}+3 k_{\|}\right) \\
c_{t}^{2} & =\frac{3 a^{2}}{8 m}\left(3 k_{\perp}+k_{\|}\right),
\end{aligned}
$$

with $m$ the mass of the points, and $a$ the lattice spacing.

Unfortunately, for purely technical reasons, it is not possible to adopt this form of the nearest neighbor force everywhere on the lattice. In order to solve this model as in the previous sections, it is necessary that mass points on opposite sides of the crack experience a force only along the line between their centers. As partial compensation, however, one can take this spring constant $k_{\|}^{I}$ to be different from the parallel force constant $k_{\|}$elsewhere in the lattice. Numerical calculations show that varying $k_{\|}^{I}$ within reasonable bounds has very little effect upon the physical results.

Taking bond interactions between neighbors in this way, one can form a correspondence with Section 3. Far ahead of the crack tip, the ratio of vertical displacements of points right above the crack line to vertical displacement at the top of the strip is

$$
Q_{0}=\frac{1 / 4\left(k_{\perp}+3 k_{\|}\right)}{1 / 4\left(k_{\perp}+3 k_{\|}\right)+2 N(3 / 4) k_{\|}^{I}} .
$$

As a fracture criterion, one demands that bonds snap when the distance between neighbors across the crack line is $2 u_{f}$ greater than it is in equilibrium, so that the energy needed for fracture is

$$
E_{\text {break }}=2 k_{\|}^{I} u_{f}^{2}
$$

The displacement of the top of the strip just necessary to supply enough energy to snap these bonds is given again by Eq. (3.10) and Eq. (3.11).

The main difference between the calculation here and the one in Section 3 results from a consideration of the fracture criterion. Letting $\vec{u}(0,1 / 2)$ be a point on the crack line, its bond with a neighbor on the other side will snap when

$$
\begin{gathered}
|\vec{u}(0,1 / 2)-\vec{u}(0,-1 / 2)|>2 u_{f} \\
\Rightarrow \frac{1}{2}\left[\frac{\sqrt{3}}{2}\left(u^{y}(0,1 / 2)-u^{y}(0,-1 / 2)\right)+\frac{1}{2}\left(u^{x}(0,1 / 2)-u^{x}(0,-1 / 2)\right)\right]>u_{f} .
\end{gathered}
$$

However, if one searches for the variable in terms of which Eq. (3.23) and its sequels remains true, one finds instead

$$
U=\frac{1}{2}\left[\left(u^{y}(0,1 / 2)-u^{y}(0,-1 / 2)\right)+\frac{1}{\sqrt{3}}\left(u^{x}(0,1 / 2)-u^{x}(0,-1 / 2)\right)\right]
$$




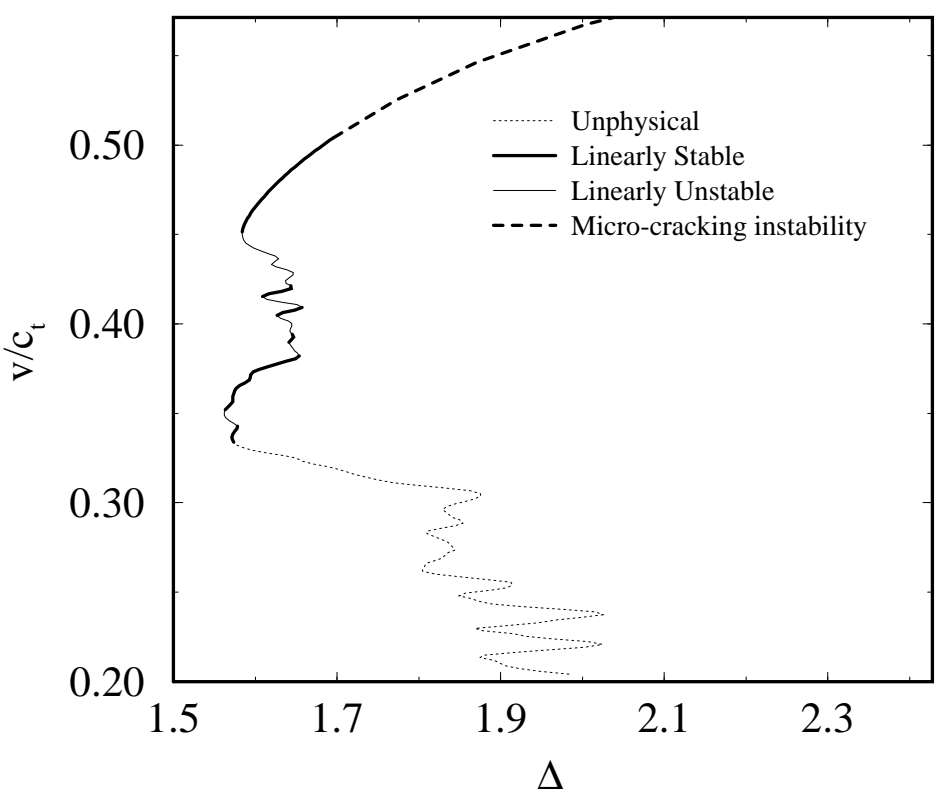

Figure 5.2: Velocity $v$ measured in units of the transverse wave speed $c_{t}$ as a function of $\Delta$, for the model of this section, calculated for $N=15, b=$ $0.012, k_{\perp}=-.3, k_{\|}=2.666, k_{\|}^{I}=2.366$. The thick lines are physically realizable states, the thin solid lines are linearly unstable, and the dotted lines are unphysical states.

is what is needed. The reason is that far ahead of the crack, $U$ defined this way approaches $u^{y}$, and all the displacements far to the right are purely vertical. In terms of this variable, fracture occurs when

$$
U(t=0)=u_{f} \frac{2}{\sqrt{3}},
$$

as claimed before Eq. (3.39). A second difference has to do with the form of the dissipation. Experimentally, waves of frequency $\omega$ decay at a rate $b(\omega) \sim \omega$. This does not seem well understood theoretically, but in any event, the calculation uses dissipation in the form

$$
i \omega b_{0} \sqrt{\omega_{0}^{2}+\omega^{2}} \vec{u}
$$

rather than $i \omega b u$ as before. The constant $\omega_{0}$ is chosen to be something smaller than frequencies of interest.

What remains is to calculate the function $Q$ appropriate to this lattice. It is a lengthy task, carried out with symbolic algebra, and relegated to Appendix VI. No really new ideas enter; some of the results are summarized in Fig. 5.2 and Table 6.1. Fig. 5.2 indicates that there are small separate bands of low-velocity states, separated by small unstable regions. Note, November 1995: Mode I fracture is subject to several instabilities beyond those studied here. The complete story has yet to be worked out. 


\section{Comparison with Experiment}

From the lattice theory emerge three predictions which can be tested experimentally.

1. For a band of velocities beginning at zero and proceeding up to around $30 \%$ of the relevant wave speed, steady crack motion is impossible.

2. Steadily moving cracks emit radiation.

3. At around $60 \%$ of the wave speed crack tips become unstable, repeatedly attempting to branch, and creating microcracks whose spacing is governed by the attenuation rate of high-frequency sound waves. The critical velocity for this instability depends on details of microstructure.

We have carried out experiments in Plexiglas (PMMA), differing from those previously reported (FINEBERG, et. al., 1991, 1992) because of improvements in instrumentation that lead to a five-fold increase in the accuracy of velocity measurements. The experiments are conducted in thin strips whose geometry is chosen to match as closely as possible conditions of the theory. Results from the various lattice models are compared with these experiments in Table 6.1. All of the phenomena seen in the lattice are present in the experiment, although the velocities at which they occur are different. Since changing from a triangular to a square lattice changes the critical velocities substantially, this discrepancy with experiment is not surprising. Plexiglas is a polymeric solid, and bears no microstructural resemblance to a triangular lattice. It is certainly possible to study more complicated lattice theories numerically in search of quantitative agreement, but in the following discussion, we want mainly to emphasize the qualitative correspondence between the experiments and the theory.

The first prediction has been verified whenever crack dynamics have been measured carefully in brittle materials (TAKAHASHI, MATSUSHIGE, AND SAKURADA 1984), although not generally given much significance. In PMMA, the jump from quasi-static to rapid motion goes to a velocity of around $175 \mathrm{~m} / \mathrm{s}$, which is $18 \%$ of the Rayleigh wave speed; three of our experimental runs at three levels of externally imposed strain are shown in Fig. 6.1. The large accelerations seen in this figure are not inconsistent with continuum theory, as one sees by comparing with Fig. 2.2. From Eq. (2.2) with $c_{l}=2000 \mathrm{~m} / \mathrm{sec}$ and $w=4 \mathrm{~cm}$, continuum theory predicts accelerations on the order $10^{8} \mathrm{~m} / \mathrm{sec}^{2}$, or $10^{2}$ $\mathrm{m} / \mathrm{sec} / \mu$-sec. However, it is difficult within a continuum framework to account for the fact that cracks can be made to initiate for such a wide range of external conditions, and that the velocity at which rapid acceleration abruptly terminates is fairly independent of the amount of energy pouring into the crack tip. From the perspective of the lattice theories, both these facts are natural. Crack motion is an activated process, and can therefore begin for a range of external conditions, while the rapid acceleration ends when the crack velocity has passed through the forbidden band.

Conceptually, one likes to think of cracks becoming unstable when their infinitesimal extension releases more energy than it consumes, so that the crack slowly accelerates up to high velocities. Unfortunately these slow extensions are dynamically forbidden. The crack must begin its dynamic life at high velocities, and the criterion for fracture initiation must be understood as a nucleation event. These remarks would seem to contradict the fact of quasi-static crack motion. In the context of the lattice models, the contradiction can be resolved if quasi-static growth is always a thermally or chemically aided process. In addition, apparent quasi-static motion may actually be a stick-slip process, in which a crack 


\begin{tabular}{|c|c|c|}
\hline Lattice & $v_{\min }$ & $v_{c}$ \\
\hline One-d $(N=9, b=0.01)$ & 0.3 & - \\
\hline Mode III, triangular $(N=9, b=0.01)$ & 0.28 & 0.67 \\
\hline Mode III, square $(N=9, b=0.01)$ & 0.36 & 0.76 \\
\hline Mode I $(N=15)$ & 0.33 & 0.56 \\
\hline Mode I $(N=30)$ & 0.33 & 0.55 \\
\hline PMMA & 0.2 & 0.33 \\
\hline
\end{tabular}

Table 6.1: Calculations of the minimum nonzero steady-state crack velocity, $v_{\min }$, and the velocity $v_{c}$ at which the branching instability begins for several lattices. In all cases, the calculations are carried out in strips $2 N+1$ lattice points hight. Mode III is the lattice of Section 4, and Mode I is the lattice of Section 5. In the One-d and Mode III cases, velocities are measured as fractions of the sound speed, while in the Mode I case, and in PMMA they are measured as fractions of the transverse wave speed. The Mode I lattice parameters are chosen to fit the Poisson ratio, and measured ultrasonic attenuation of PMMA, and are $k_{\perp}=-.3, k_{\|}=2.666, k_{\|}^{I}=2.366$; experimentally dissipation is observed to be proportional to frequency, and this scaling is used in the calculation as well, with the experimental coefficient of 0.012, (JACKSON, PENTECOST, AND POWLES, 1972) . The comparison has been made with the plane strain Poisson ratio; to compare with the plane stress Poisson ratio, one can use $k_{\perp}=0$ instead of $k_{\perp}=-.3$, but the results are not substantially different.

alternates between rapid motion and arrest on scales too fast for ordinary measurements to detect. This observation may have implications for fracture testing. Recent measurements of the fracture energy of PMMA have all been in the range of 200 to $350 \mathrm{~J} / \mathrm{m}^{2}$ (KATSAMANIS AND DELIDES, 1988) . Our experiments involve long center edge cracks in long strips, so that the fracture energy can be deduced simply by measuring the stress per area in the strip. Inspection of Fig. 6.1 shows that the fracture energy of our samples can be as low as 80 $\mathrm{J} / \mathrm{m}^{2}$ - three times less than typical previous measurements. Note, November, 1995: These low fracture energies have not been reproduced in subsequent experiments, and this claim is incorrect. Fracture energies are consistent with previous measurements. One gets a sense of how close one is to the minimum fracture energy by watching the rate of acceleration after the crack initiates. We suspect that many of the experimental techniques used to measure fracture energies involve dynamical effects at a more important level than has been appreciated, but this point requires additional investigation.

The second of the predictions has not really been verified experimentally. It is true that moving cracks always emit sound (GROSS, et. al., 1993). However, acoustic transducers detect only up to around $10 \mathrm{MHz}$, which is far below the frequencies at which atomic bonds snap when a crack moves at hundreds or thousands of meters per second. At such high frequencies, one should expect sound to thermalize rapidly, and manifest itself as heat. Certainly there is always a large temperature rise near crack tips (GREEN AND 


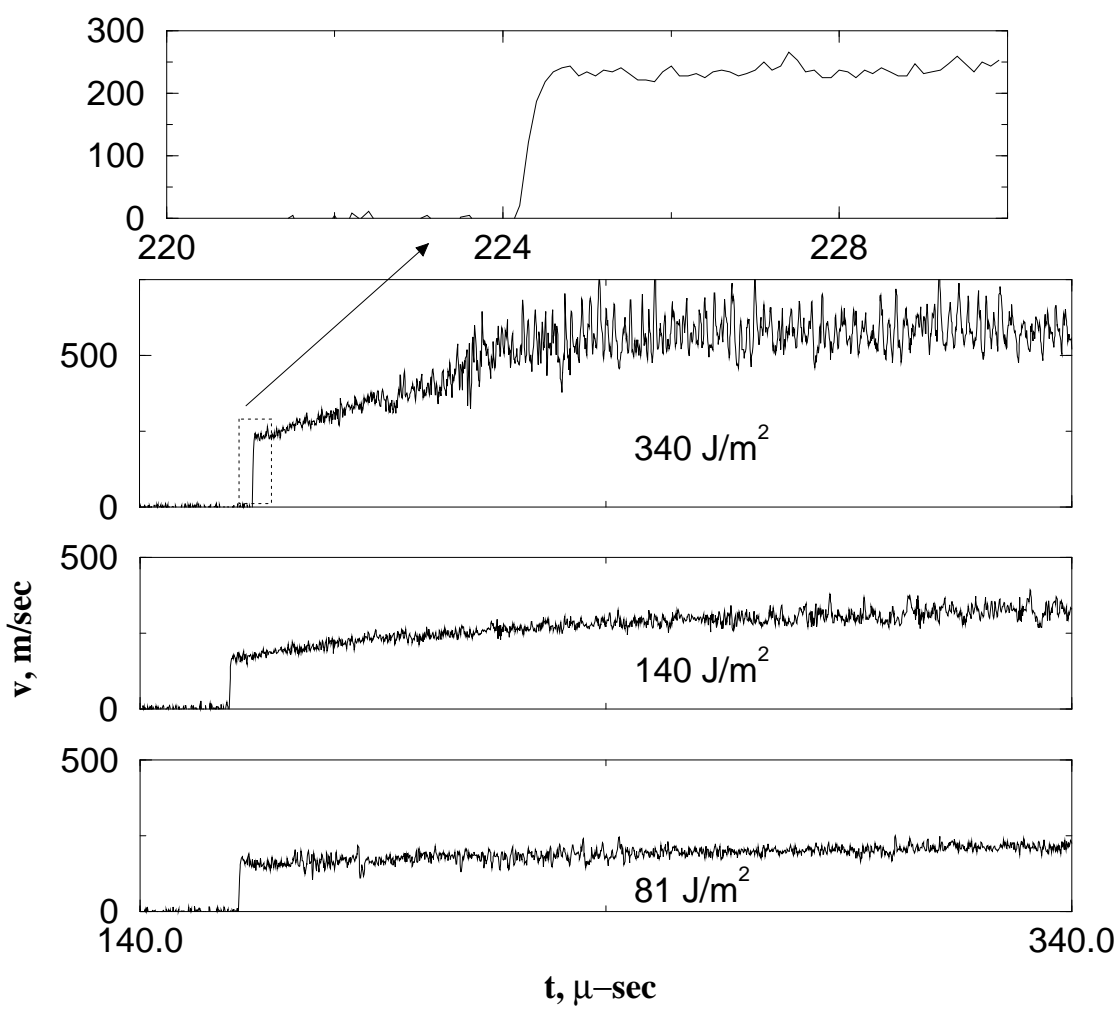

Figure 6.1: Three measurements of velocity versus time in Plexiglas (PMMA). The experiments are conducted in strips, and the energy stored per unit area to the right of the crack is printed each picture. Cracks are made to initiate with different energies available per length by preparing the notch which initiates fracture in different ways. Even if the system is stressed so gently that the crack does not accelerate noticeably after it begins rapid motion, there is still a jump in velocity up to around 200 $\mathrm{m} / \mathrm{s}$. The upper part of the figure should be compared with Fig. 2.2. The fracture energy of the sample depicted in the lower part of the figure is several times lower than any other value for PMMA in the literature; see Katsamanis and Delides, (1988).

PRATT, 1974; FULLER, FOX, AND FIELD, 1983), but there are many potential sources for it, especially plastic deformation. To the extent that the high-frequency sound decays within the core region surrounding the crack tip, it causes no conceptual difficulty for the continuum picture of fracture. Our prediction is that the size of the heated zone should be set by the dissipation coefficient $b$, but this estimate has not been tested experimentally.

The third of the predictions corresponds to observations in PMMA, which observe the emergence of microcracks after about $40 \%$ of the Rayleigh wave speed. The first publication we are aware of describing these as an important factor in the fracture of PMMA is by Doyle (1983); their role has also been emphasized by Ravi-Chandar and Knauss (1984). Our use of thin strip samples enables us to obtain cracks traveling in steady state at different mean velocities, and locate the onset of micro-cracking with greater precision. A drawing 
of these cracks soon after onset is displayed in Fig. 6.2. We can use Eq. (4.47) to estimate the spacing of these branches.

The main experimental ingredient needed for the estimate is acoustic attenuation at high frequencies, which has been measured in PMMA by Jackson, Pentecost, and Powles (1972). They find that attenuation per cycle is nearly constant in PMMA as a function of frequency and temperature, and obeys

$$
\alpha \lambda=0.02-0.07
$$

where $\alpha$ is attenuation per length, and $\lambda$ is the wavelength of the waves one wants to consider (the experimental measurements are for longitudinal waves.) The lower value of 0.02 holds up to $100 \mathrm{MHz}$; there is a gap in the measurements, and 0.07 is measured at 10 GHz. Taking $\alpha \lambda=0.02$, one has that attenuation per time $b$ is given by $b \lambda / c_{l}=0.02$, with $c_{l}$ the longitudinal sound speed. Polymer units of size $0.5 \mu$ separate coherently in PMMA so PMMA will be treated as a lattice of masses separated by $a=0.5 \mu \mathrm{m}$. Now the waves being excited by the passage of the crack have wavelength $\lambda=a c_{l} / v$, where $a=0.5 \mu \mathrm{m}$ is the lattice spacing and $v$ is the crack velocity, so $b \sim 0.02 v / a \sim 12 \mathrm{MHz}$, for $a \sim 0.5 \mu \mathrm{m}$ and $v \sim 300 \mathrm{~m} / \mathrm{sec}$. Taking $\left[\partial u_{h} / \partial \Delta\right]\left(\Delta-\Delta_{c}\right) / \delta u_{h}$ to be on the order of $10 \%$, one has from Eq. (4.47) and Eq. (6.1) the crude estimate that microcracks appear with a frequency of

$$
6 \mathrm{MHz} \Rightarrow \text { Spacing }=0.05 \mathrm{~mm}
$$

for a crack traveling at $300 \mathrm{~m} / \mathrm{sec}$. Close examination of the 1/32 inch samples of PMMA reveals a comb of micro-cracks, shown in Fig. 6.2, resembling those in Fig. 4.7, and extending into the surface with a typical separation of about $0.07 \mathrm{~mm}$. This spacing is larger than the rough theoretical estimate, but of the same order of magnitude. Both in the simulations and in the experiment, microcracks on the upper and lower portions of the surface are staggered.

There is also experimental evidence for the claim that the onset of the microcracking instability is sensitive to details of lattice structure. As shown in Table 6.1, moving from a triangular to a square lattice pushes onset of the instability to higher velocities; the general lesson should be that cleavage along weak lattice planes discourages micro-cracking. Indeed, cracks reaching the transverse wave speed (and in one case claimed to exceed it) have been measured in cleavage of LiFl (GILMAN, KNUDSEN, AND WALSH, 1958), while Cotterell (1964) has measured speeds approaching the Rayleigh wave speed for cracks traveling along prescribed grooves in PMMA.

The comparison between simulation and experiment has to be made with two qualifications. First, the simulations are on strips only nine atoms high, from all points of view much smaller than the experiments. Second, the experiments are clearly not twodimensional. The cracks seen in Fig. 6.2 are those that were visible within a particular plane, about $100 \mu$ thick. Changing the vertical plane changes the details of the pattern of microcracks; this observation partly explains why the microcracks in the figure appear intermittent.

In connection with this three-dimensional structure, we note that Perrin and Rice (1994) have recently found an interesting implication of elastic theory. When a crack front is considered in three dimensions, it is linearly stable against local fluctuations in fracture toughness, but upon repeated contact with inhomogeneities, the crack front can deform 


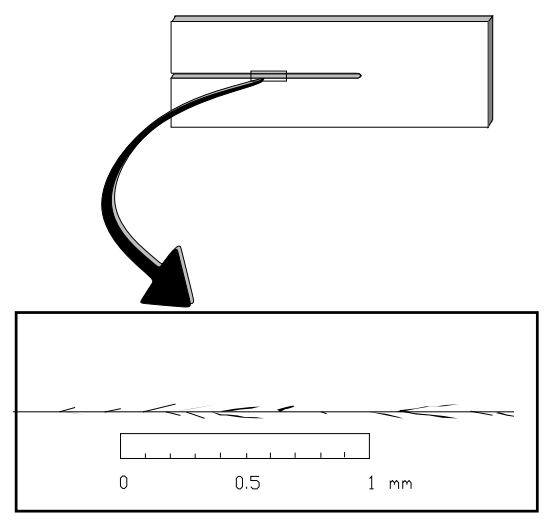

Figure 6.2: Drawing of small microcracks that begin to emerge from a crack after it passes a velocity of around $330 \mathrm{~m} / \mathrm{sec}$ in Plexiglas (PMMA). The basic geometry is the same as that shown in Fig. 4.7, with a spacing on the order of $0.07 \mathrm{~mm}$. This diagram shows the microcracks near the point where they first appear; later, they are longer and more densely packed. The microcracks shown in the figure are those that appear in a microscope whose depth of field is about $100 \mu$, and the pattern of microcracks changes as one looks at different vertical planes by changing the focus of the microscope.

considerably, and experience velocity fluctuations on the order of a quarter of the Rayleigh wave speed. Therefore, a crack moving nominally at one-third the wave speed may locally be moving much faster (or much slower). This picture provides one possible explanation for the fact that microcracks appear experimentally at velocities noticeably lower than that predicted by the lattice models.

In prior publications (FINEBERG et. al., 1991, 1992), our group has described a $500 \mathrm{KHz}$ frequency emitted from PMMA, corresponding to a $1 \mathrm{~mm}$ scale structure on the surface. This spatial scale is several times larger than the one we have attributed to the onset of microcracking; the $1 \mathrm{~mm}$ oscillations begin in earnest at a somewhat higher velocity (400 $\mathrm{m} / \mathrm{sec}$ ) than the initial microcracks $(330 \mathrm{~m} / \mathrm{sec})$, and correspond to bunches of especially dense microcracks grouped at roughly $1 \mathrm{~mm}$ intervals. We do not yet know whether this phenomenon is the natural evolution of the microcracking instability when driven to large amplitudes, or whether new physical processes are coming into play. Thus the original oscillatory instability with which we began our investigations is still not well understood.

While resolving numerous questions that arise in continuum models, and providing a qualitative understanding for many features of the experiments, the lattice models raise as many questions as they answer. When should one get micro-cracks, and when dislocations? How do dynamic ductile-brittle transitions occur? What are the effects of thermal noise? How is quasi-static motion possible? What would happen in a random lattice? How should one treat a polymer? How can the results be generalized to three dimensions? How does the instability progress towards macroscopic branching? What happens in larger-scale simulations with realistic bond forces? What are the implications for fracture testing? These are some of the points that deserver further investigation. 
This work was supported in part by the Texas Advanced Research Program, grant number 3658-002. These topics were discussed with many of the participants in an UCSBITP workshop on Spatially Extended Nonequilibrium Systems, particularly J. S. Langer, and M. Barber, and are the result of a collaboration with experimentalists at the Center for Nonlinear Dynamics at UT Austin, including H. Swinney, J. Fineberg, J. Urbach, and W. McCormick. Special thanks are due to J. Urbach for assistance with electronics in velocity measurements.

\section{References}

ASHURST, W. T., and HOOVER, W. G.(1976) Microscopic fracture studies in the twodimensional triangular lattice, Phys. Rev. B14, 1465-1473.

ATKINSON, W., and CABRERA, N.(1965) Motion of a Frenkel-Kontorova dislocation in a one-dimensional crystal, Phys. Rev. 138, A763-A766

BergkVist, H. (1973) The motion of a brittle crack, J. Mech. Phys. Solids 21, 229-239.

BERGKVIST, H. (1974) Some experiments on crack motion and arrest in polymethylmethacrylate, Engng. Fracture Mech. 6, 621-626.

CELLI, V., and FLYTZANIS, N.(1970) Motion of a screw dislocation in a crystal, J. Appl. Phys. 41, 4443-4447.

COTTERELl, B. (1965) Velocity effects in fracture propagation, Appl. Mater. Res. 4 $227-232$.

DÖLL, W., and WEIDMANN, G. W.(1976) Transition from slow to fast crack propagation in PMMA, J. Mater. Sci. Lett. 11, 2348-2350.

DOYLE, M. (1983) A mechanism of crack branching in polymethylmethacrylate and the origin of the bands on the surfaces of fracture, J. Mater. Sci. 18, 687-702.

FINEBERG, J., GROSS, S., MARDER, M. and SWINNEY, H., et. al.(1991) Instability in dynamic fracture, Phys. Rev. Lett. 67, 141-144.

FINEBERG, J., GROSS, S., MARDER, M. and SWINNEY, H., et. al. (1992) Instability in the propagation of fast cracks, Phys. Rev. B45, 5146-5154.

FREUND, L. B.(1974)Crack propagation in an elastic solid subjected to general loading. IV. Obliquely incident stress pulse, J. Mech. Phys. Solids 22, 137-146.

FREUND, L. B. (1990)Dynamic Fracture Mechanics Cambridge University Press, New York.

FULLER, K. N. G., FOX, P. G. , and FIELD, J. E. (1975) The temperature rise at the tip of fast-moving cracks in glassy polymers, Proc. $R$. Soc. Lond. A 341, 537-557.

GAO, H. (1993) Surface roughening and branching instabilities in dynamic fracture, J. Mech. Phys. Solids 41, 457-486.

GILMAN, J. J., KNUDSEN, C., and WALSH, W. P. (1958) Cleavage cracks and dislocation in LiF crystals, J. Appl. Phys 29, 601-607.

GRADSHTEYN I. S. and RYZHIK, I. M. (1980) Table of integrals, series and products. Academic Press, New York.

GREEN, A. K., and PRATT, P. L.(1974) Measurement of the dynamic fracture toughness of polymethylmethacrylate by high-speed photography, Engng. Fracture Mech. 6, 71-80.

GROSS, S., FINEBERG, J., MCCORMICK, W. D., MARDER, M., and SWINNEY, H. (1993) Acoustic emissions from rapidly moving cracks, Phys. Rev. Lett. 71, 3162-3165. 
JACKSOn, D. A., PEnTECOST, H. T. A., and POWLES, J. G. (1972) Hypersonic absorption in amorphous polymers by light scattering, Mol. Phys. 23, 425-432.

KANNINEN, M. F., and POPELAR, C.(1985) Advanced Fracture Mechanics Oxford University Press, New York.

KATSAMANIS, F. G., and DELIDES, C. G.(1988) Fracture surface energy measurements of PMMA: a new experimental approach, J. Phys. D 21, 79-86.

KNAUSS, W. G.(1966) Stresses in an infinite strip containing a semi-infinite crack, J. Appl. Mech. 33, 356-362.

KOBAYASHI, A., OHTANI, N., and SATO, T. (1974) Phenomenological aspects of viscoelastic crack propagation, J. Appl. Polymer Sci. 18, 1625-1638.

KUlAKHMETOVA, SH. A., SARAIKIN, V. A., and SLEPYAN, L. I. (1984) Plane problem of a crack in a lattice, Mechanics of Solids 19, 101-108.

LANGER, J. S. (1993) Dynamic model of onset and propagation of fracture, Phys. Rev. Lett. 70, 3592-3594.

LANGER, J. S., and NAKANISHI, H. (1993) Models of crack propagation. II. Twodimensional model with dissipation on the fracture surface, Phys. Rev. E48, 439-448.

LIU, X. (1993) Dynamics of fracture propagation, (Dissertation, University of Texas)

LIU, X., and MARDER, M.(1991) The energy of a steady-state crack in a strip, J. Mech. Phys. Sol 39, 947-961.

MACHOVÁ, A. (1992) Molecular dynamic simulation of microcrack initiation, Materials Science and Engineering A149 153-165.

Manneville, P. (1990) Dissipative Structures and Weak Turbulence, Academic Press, Boston Chapter 6, section 6

MARDER, M. (1991) New dynamical equation for cracks, Phys. Rev. Lett. 66, 24842487.

MARDER, M., and LIU, X. (1993) Instability in lattice fracture, Phys. Rev. Lett. 71, 2417-2420.

MECHOLSKY, J. J. (1985) Fracture analysis of glass surfaces, in Strength of Inorganic Glass, (C. R.KURKJIAN, ed.), pp. 569-590. Plenum Press, New York.

NOBLE, B. (1959) Methods Based on the Wiener-Hopf Technique for the Solution of Partial Differential Equations Pergamon Press, New York.

PERRIN, G., and RICE, J. R. (1994), Disordering of a dynamic planar crack front in a model elastic medium of randomly variable toughness, J. Mech. Phys. Solids 42 1047-1064.

RAVI-CHANDAR, K. , and KNAUSS, W. G. (1984) An experimental investigation into dynamic fracture: III. On steady state crack propagation and crack branching, Int. J. Fracture 26 , 141-154.

RICE, J. R., BEN-ZION, Y., and KIM, K. (1994) Three-dimensional perturbative solution for a dynamic planar crack moving unsteadily in a model elastic solid, J. Mech. Phys. Solids 42, 813-843.

SIERADZKI, K., DIENES, G. J., PASKIN, A., and MASSOUMZADEH, B., (1989) Atomistics of crack branching, Acta. Metall. 36, 651

SLEPYAN, L. I. (1981) Dynamics of brittle fracture in lattice, Doklady Soviet Phys. 26 538-540.

SLEPYAN, L. I.(1992) Principle of maximum energy dissipation rate in crack dynamics, Doklady Soviet Phys. 41, 1019-1033. 
SLEPYAN, L. I.(1993) Principle of maximum energy dissipation rate in crack dynamics, J. Mech. Phys. Solids 41, 1019-1033.

TAKAHASHI, K., MATSUSHIGE, K. and SAKURADA, Y. , (1984) Precise evaluation of fast fracture velocities in acrylic polymers at the slow-to-fast transition, J. Mater. Sci. 19, 4026-4034.

THOMson, R. (1986) Physics of fracture, Solid State Physics 39, 1-129.

THOMSON, R., HSIEH, C., and RANA, V.(1971) Lattice trapping of fracture cracks, J. Appl. Phys. 42, 3154-3160.

WASHABAUGH, P. D., and KNAUSS, W. G.(1993) Non-steady periodic behavior in the dynamic fracture of PMMA, Int. J. Fracture 59, 189-197.

WILLIS, J. R. (1990) Accelerating cracks and related problems, , in Elasticity: Mathematical Methods and Applications (G. EASON, AND R. W. OGDEN, eds.) pp. 397-409. Halston Press, New York.

XU, Y., and KEER, L. M.(1992) Non-planar deviation of an initially straight moving crack, Engng. Fracture Mech. 41, 577-585.

YOFFE, E. H.(1951) The moving Griffith crack, Phil. Mag. 42, 739-750.

ZHOU, S. J., CARLSSON, A. E., and THOMSON, R.(1994) Crack blunting effects on dislocation emission from cracks, Phys. Rev. Lett. 72, 852-855. 


\section{Appendix I.}

\section{Calculation of Lattice-Trapped States}

In this Appendix, we will calculate the static solutions of Eq. (3.1). Dropping the subscript + , one begins with the equation

$$
0=u_{m+1}-2 u_{m}+u_{m-1}+\frac{1}{N}\left(U_{N}-u_{m}\right)-2 u_{m} \theta_{m}
$$

where

$$
\theta_{m}=\left\{\begin{array}{ll}
1 & \text { for } m \geq 0 \\
0 & \text { for } m<0
\end{array} .\right.
$$

For this solution to follow from Eq. (3.1), one must have that

$$
u_{0}<1 \text { and } u_{-1}>1 \text {. }
$$

In other words, the bond at zero has not snapped, so it must be stretched less than a distance of 1 , but the bond at -1 has snapped, so it must be stretched more than 1 . The solution of Eq. (I.1) is

$$
u_{m}=\left\{\begin{array}{ll}
u_{r}(m)=\frac{U_{N}}{2 N+1}+u_{r} y_{r}^{m} & \text { for } m \geq 0 \\
u_{l}(m)=U_{N}+u_{l} y_{l}^{m} & \text { for } m<0
\end{array},\right.
$$

where

$$
y_{l}-2+1 / y_{l}-1 / N=0
$$

and

$$
y_{r}-2+1 / y_{r}-2-1 / N=0 .
$$

In order for the left and right solutions to match onto one another smoothly, one must demand that

$$
u_{l}(0)=u_{r}(0) \text { and } u_{l}(-1)=u_{r}(-1) .
$$

The four equations Eq. (I.5) and Eq. (I.6) determine $u_{l}, u_{r}, y_{l}$ and $y_{r}$ as functions of $U_{N}$. Using $U_{c}=\sqrt{2 N+1}$, turning the condition Eq. (I.2) into a condition on $\Delta$, and working in the limit $N \rightarrow \infty$ gives

$$
\Delta<\frac{\sqrt{3}+1}{\sqrt{2}} \text { for } u_{0}<1
$$

and

$$
\Delta>\frac{\sqrt{3}-1}{\sqrt{2}} \text { for } u_{-1}>1
$$

These are the boundaries for the region of lattice trapping. 


\section{Appendix II.}

Evaluation of $\Delta$ for small and for large $v$

In this Appendix, we will evaluate $\Delta(v)$ for the one-dimensional model in the limits $v \rightarrow 0$ and $v \rightarrow 1$, starting from Eq. (3.40)

It is possible to evaluate the product

$$
P(A)=\prod \frac{w_{i}^{+}}{w_{i}^{-}},
$$

where for generality $w_{i}$ is the root of

$$
\omega^{2}-4 \sin ^{2}(\omega / 2 v)-4 A^{2}
$$

analytically in certain limits. In the limit of low velocity, it is helpful first to write the condition that Eq. (II.2) have a root as

$$
\omega=2 v \sin ^{-1} \sqrt{\omega^{2} / 4-A^{2}} .
$$

In the limit of low velocity, by looking at a graph of Eq. (II.2), one sees that the roots are given approximately by

$$
\begin{aligned}
& w_{i}^{+}=2 v\left[\left(n_{0}+i\right) \pi-\sin ^{-1}\left(\sqrt{v^{2}\left[n_{0}+i\right]^{2} \pi^{2}-A^{2}}\right)\right], \\
& w_{i}^{-}=2 v\left[\left(n_{0}+i\right) \pi+\sin ^{-1}\left(\sqrt{v^{2}\left[n_{0}+i\right]^{2} \pi^{2}-A^{2}}\right)\right],
\end{aligned}
$$

and that there is always one more positive root $w_{i}^{+}$than there are positive roots $w_{i}^{-}$. The starting integer $n_{0}$ is given by

$$
A=2 v n_{0} \pi
$$

(one will have to fiddle around with $v$ a bit to make this precisely true) and the largest value of $i$ is $n_{f}$, given by

$$
\sqrt{A^{2}+1}=\left(n_{0}+n_{f}\right) v \pi
$$

(this comes close to the truth in the limit of small $v$ ).

For the moment, let us restrict ourselves just to the positive roots of Eq. (II.2). Then

$$
\begin{aligned}
& \frac{1}{2} \ln P(A)=\sum_{i=0}^{n_{f}-1} \ln \left[\frac{\left(n_{0}+i\right) \pi-\sin ^{-1}\left(\sqrt{v^{2}\left[n_{0}+i\right]^{2} \pi^{2}-A^{2}}\right)}{\left(n_{0}+i\right) \pi+\sin ^{-1}\left(\sqrt{v^{2}\left[n_{0}+i\right]^{2} \pi^{2}-A^{2}}\right)}\right]+\ln \left[\left(n_{0}+n_{f}\right) \pi\right] \\
& =\sum_{i=0}^{n_{f}-1} \ln \left[\frac{1-\sin ^{-1}\left(\sqrt{v^{2}\left[n_{0}+i\right]^{2} \pi^{2}-A^{2}}\right) /\left(n_{0}+i\right) \pi}{1+\sin ^{-1}\left(\sqrt{v^{2}\left[n_{0}+i\right]^{2} \pi^{2}-A^{2}}\right) /\left(n_{0}+i\right) \pi}\right]+\ln \left[\left(n_{0}+n_{f}\right) \pi\right]
\end{aligned}
$$




$$
\begin{gathered}
=\sum_{i=0}^{n_{f}-1}(-2) \sin ^{-1}\left(\sqrt{v^{2}\left[n_{0}+i\right]^{2} \pi^{2}-A^{2}}\right) /\left(n_{0}+i\right) \pi+\ln \left[\left(n_{0}+n_{f}\right) \pi\right] \\
=\int_{A}^{\sqrt{A^{2}+1}} d x\left(\frac{-2}{\pi}\right) \frac{\sin ^{-1} \sqrt{x^{2}-A^{2}}}{x}+\ln \left(\sqrt{A^{2}+1} / v\right)
\end{gathered}
$$

and integrating by parts gives

$$
\frac{1}{2} \ln P(A)=\ln \frac{1}{v}+\frac{2}{\pi} \int_{A}^{\sqrt{A^{2}+1}} \frac{x \ln x d x}{\sqrt{x^{2}-A^{2}} \sqrt{1+A^{2}-x^{2}}}
$$

which after the change of variables $x^{2}=A^{2}+\sin ^{2} \theta$ becomes

$$
P(A)=\frac{1}{v^{2}} \exp \left[\frac{1}{\pi} \int_{0}^{\pi} \ln \left[A^{2}+\sin ^{2} \theta\right]\right] d \theta .
$$

In the particular case of Eq. (3.40), one wants to evaluate

$$
(\Delta)^{2}=\frac{P\left(\sqrt{1+\epsilon^{2}} / 2\right)}{P(\epsilon / 2)}=\exp \left[\frac{1}{\pi} \int_{0}^{\pi} \ln \left[\frac{\epsilon^{2} / 4+1 / 4+\sin ^{2} \theta}{\epsilon^{2} / 4+\sin ^{2} \theta}\right]\right] d \theta
$$

Gradshteyn and Rizhyk (1980) have the integral (4.399)

$$
\int_{0}^{\pi} d x \ln \left(1+a \sin ^{2} x\right)=2 \pi \ln \left(\frac{1+\sqrt{1+a}}{2}\right) .
$$

This gives finally

$$
\Delta=\frac{\sqrt{\epsilon^{2} / 4+1 / 4}+\sqrt{1+\epsilon^{2} / 4+1 / 4}}{\epsilon / 2+\sqrt{1+\epsilon^{2} / 4}} .
$$

In the limit $\epsilon \rightarrow 0$, one has that

$$
\Delta=\frac{1+\sqrt{5}}{2}=1.6180 \ldots
$$

the golden mean, in agreement to three places with the direct evaluation of the roots in Eq. (3.40). One has that a stationary lattice crack in a noiseless environment will not begin to move until the driving strain exceeds by this amount the strain that would be predicted in a continuum model.

At velocities that approach 1 , there is one root $f^{+}$and one root $g^{+}$. In the limit of small $\epsilon$, and for $v=1, g^{+}=1.91892$. One finds $f^{+}=\sqrt{2 \sqrt{3} \epsilon+12(1-v)}$. Therefore

$$
\Delta \rightarrow \sqrt{\frac{1.91892}{\sqrt{2 \sqrt{3} \epsilon+12(1-v)}}}
$$




\section{Appendix III. Calculation of $\Delta$ for small damping}

This Appendix describes the calculation of $\Delta(v)$ in the limit of zero damping, $b=0$ for the triangular lattice of Section 3. Begin with Eq. (3.40).

In order to make use of this expression, one must find an efficient way to locate the roots and poles of $Q$. This task is equivalent to finding the roots of $F$, defined in Eq. (4.23), and the roots of

$$
G(\omega) \equiv F(\omega)-1-\cos (\omega / 2 v),
$$

which is the denominator of $Q$. Both $F$ and $G$ have poles, but at the same values of $\omega$, and these poles cancel. The roots fall into two classes. In the first class are roots for which $|z|>1$. These roots are well separated, and can be located by a standard root-finding algorithm. In the second class are roots for which $|z| \leq 1$. These roots are very finely spaced, and for each region where $|z|<1$, there are of order $N$ roots. These may be located by looking for the values of $y$ where $F$ vanishes. These are denoted by $y^{F}$, and are indexed by $j$, which varies from 1 to $N$. Inverting $F$ to find $y$ one has

$$
y_{j}^{F}=e^{i(2 j-1) \pi /(2 N+1)+\ln \left[\frac{y_{j}^{F}-\cos (\omega / 2 v)}{y_{j}^{F} \cos (\omega / 2 v)-1}\right] /(2 N+1)} .
$$

Therefore

$$
z_{j}^{F}=\cos \left[\frac{(2 j-1) \pi}{2 N+1}+\frac{1}{i(2 N+1)} \ln \left\{\frac{y_{j}^{F}-\cos \omega / 2 v}{y_{j}^{F}(\cos \omega / 2 v)-1}\right\}\right]
$$

Similarly, inverting $G$ to find $y$ and $z$ one gets

$$
z_{j}^{G}=\cos \left[\frac{2 j \pi}{2 N+1}\right],
$$

The roots of $G$ are therefore very easy to find. By finding solutions of Eq. (III.3a) one finds the roots of $F$. Once all the roots are located, one determines the sign of their imaginary part for infinitesimal $b$, and finally inserts them into Eq. (3.40) to find $\Delta$. 


\section{Appendix IV. Linear stability of steady state solutions}

This Appendix carries out the linear stability analysis of steady state solutions for the Mode III model in detail. To begin, it is useful to recall the symmetries of the steady state solutions. These are

$$
u^{0}(m, n, t)=u^{0}(m+1, n, t+1 / v)
$$

and also

$$
u^{0}(m, n, t)=-u^{0}\left(m,-n, t-\left[1 / 2-g_{n}\right] / v\right) .
$$

Therefore the perturbations can be taken first to be eigenfunctions of the operator which translates $m$ by 1 , and $t$ simultaneously by $1 / v$, perturbations with time dependence of the form $e^{q t} f(n, t-m / v)$. Second, the perturbations are eigenfunctions of the operator which inverts states around the $x$ axis while moving $t$ forward by $1 / 2 v$. If this operation is repeated twice, it simply produces one of the translations of the first operator, and must therefore have eigenvalue $e^{q t}$. The operation carried out only once must therefore have eigenvalue $\pm e^{q t / 2}$; even and odd modes, associated with the inversion symmetry.

Therefore the states will be of the form

$$
u^{0}(n, t-m / v)+u^{1}(n, t-m / v) e^{q t},
$$

with $u^{1}$ small, and with both even and odd inversion symmetries possible.

Placing this state into Eq. (4.1a) and expanding to first order, one has that

$$
\begin{aligned}
& q^{2} u^{1}(m, n)+2 q \dot{u}^{1}(m, n)+\ddot{u}^{1}(m, n)= \\
& \quad \frac{1}{2}\left[\begin{array}{c}
+u^{1}\left(m+g_{n+1}-1, n+1\right)+u^{1}\left(m+g_{n+1}, n+1\right) \\
+u^{1}(m-1, n)-6 u^{1}(m, n)+u^{1}(m+1, n) \\
+u^{1}\left(m+g_{n-1}-1, n-1\right)+u^{1}\left(m+g_{n-1}, n-1\right)
\end{array}\right] \\
& -b \dot{u}^{1}(m, n)
\end{aligned}
$$

if $n>1 / 2$, taking $u_{f}=1$

$$
\begin{aligned}
& q^{2} u^{1}(m, n)+2 q \dot{u}^{1}(m, n)+\ddot{u}^{1}(m, n)= \\
& \frac{1}{2}\left[\begin{array}{c}
+u^{1}\left(m+g_{n+1}-1, n+1\right)+u^{1}\left(m+g_{n+1}, n+1\right) \\
+u^{1}(m-1, n)-4 u^{1}(m, n)+u^{1}(m+1, n) \\
+\left[u^{1}(m, n-1)-u^{1}(m, n)\right]\left[\theta(-t)-\delta\left(1-u^{0}(t)\right)\right] \\
+\left[u^{1}(m+1, n-1)-u^{1}(m, n)\right]\left[\theta(1 / 2 v-t)-\delta\left(1-u^{0}(t-1 / 2 v)\right)\right]
\end{array}\right] \\
& -b \dot{u}^{1}(m, n)
\end{aligned}
$$

if $n=1 / 2$. The boundary condition is that $u^{1}$ vanish at $n= \pm(N+1 / 2)$, and $u^{0}$ is defined by Eq. (4.19) as the difference between two mass points above and below the crack line in the steady state. Upon Fourier transforming Eq. (IV.3) one sees that it differs from the 
solution of Eq. (4.1) only in two ways. First, $-\omega^{2} \rightarrow(q-i \omega)^{2}$, changing the definition of $z$ in Eq. (4.15). Second, the driving force on the crack face is no longer the applied stress $\sigma_{\infty}$, but is now a set of two delta functions appearing near the end of Eq. (IV.3b).

Fourier transforming Eq. (IV.3) one has therefore that

$$
\begin{gathered}
z_{q}=\frac{3-\cos (\omega / v)+(q-i \omega)^{2}-i b \omega}{2 \cos (\omega / 2 v)} \\
y_{q}=z_{q}+\sqrt{z_{q}^{2}-1}
\end{gathered}
$$

and

$$
\begin{aligned}
& u_{1 / 2}^{1}(\omega) F_{q}(\omega) \\
& -\frac{1}{2} \int d t e^{i \omega t}\left\{\left[u_{-1 / 2}^{1}(t)-u_{1 / 2}^{1}(t)\right] \theta(-t)+\left[u_{-1 / 2}^{1}(t-1 / v)-u_{1 / 2}^{1}(t)\right] \theta(1 / 2 v-t)\right\} \\
=- & \frac{\left[u_{-1 / 2}^{1}(0)-u_{1 / 2}^{1}(0)\right]+e^{i \omega / 2 v}\left[u_{-1 / 2}^{1}(-1 / 2 v)-u_{1 / 2}^{1}(1 / 2 v)\right]}{2 \dot{u}^{0}(0)}
\end{aligned}
$$

with

$$
F_{q}(\omega)=\left\{\frac{y_{q}^{[N-1]}-y_{q}^{-[N-1]}}{y_{q}^{N}-y_{q}^{-N}}-2 z_{q}\right\} \cos (\omega / 2 v)+1
$$

At this point, one needs to distinguish between the modes which are odd and those which are even under inversion. Define

$$
U(t)=\frac{u_{1 / 2}^{1}(t)-u_{-1 / 2}^{1}(t)}{2}
$$

for the modes such that

$$
u_{1 / 2}^{1}(t)=-u_{-1 / 2}^{1}(t-1 / 2 v),
$$

and

$$
V(t)=\frac{u_{1 / 2}^{1}(t)-u_{-1 / 2}^{1}(t)}{2}
$$

for the modes such that

$$
u_{1 / 2}^{1}(t)=u_{-1 / 2}^{1}(t-1 / 2 v),
$$

The least stable mode is of the type $U(t)$, which will therefore be of interest in what follows.

One has that

$$
U(\omega) F_{q}-(1+\cos \omega / 2 v) U^{-}=-(1+\cos \omega / 2 v) \frac{U(t=0)}{\dot{u}^{0}(0)}
$$

or

$$
U^{+} Q_{q}+U^{-}=\left(1-Q_{q}\right) \frac{U(t=0)}{\dot{u}^{0}(0)}
$$


with $Q_{q}$ given by Eq. (4.28); the subscripts $q$ are meant to remind that $z$ depends upon $q$. This may be rewritten

$$
U^{+} \frac{1}{Q_{q}^{+}}+U^{-} \frac{1}{Q_{q}^{-}}=\left(\frac{1}{Q_{q}^{-}}-\frac{1}{Q_{q}^{+}}\right) \frac{U(t=0)}{\dot{u}^{0}(0)} .
$$

This expression can be decomposed immediately to give

$$
U^{-}(\omega) \frac{1}{Q_{q}^{-}(\omega)}=\left(\frac{1}{Q_{q}^{-}}-\frac{1}{Q_{q}^{-}(\infty)}\right) \frac{U(t=0)}{\dot{u}^{0}(0)}
$$

where a constant term has been included to avoid a delta function singularity in $U(t)$ at $t=0$. Since as before $Q_{q}^{-}(\omega)$ goes to one at when $\omega$ goes to infinity

$$
U^{-}(\omega)=\left(1-Q_{q}^{-}\right) \frac{U(t=0)}{\dot{u}^{0}(0)} .
$$

Because of time translation invariance, one expects there to be a zero mode, whose eigenfunction is $\dot{u}^{0}(t)$. By comparison with Eq. (3.28), one sees immediately that this is the case, since $Q_{q=0}$ appearing in Eq. ( $\left.I V .13\right)$ is identical to the function $Q$ defined previously by Eq. (4.28). The general consistency condition which tells which values of $q$ are allowed is

$$
\begin{gathered}
U^{-}(t=0)=\lim _{\epsilon \rightarrow 0} \int \frac{d \omega}{2 \pi} e^{i \omega \epsilon}\left(1-Q_{q}^{-}(\omega)\right) \frac{U(t=0)}{\dot{u}^{0}(0)} \\
\Rightarrow \dot{u}^{0}(0)=\int \frac{d \omega}{2 \pi}\left(1-Q_{q}^{-}(\omega)\right) .
\end{gathered}
$$

Using the fact that that the zero mode is just $\dot{u}^{0}$, one can also write

$$
0=\int \frac{d \omega}{2 \pi}\left(Q_{0}^{-}(\omega)-Q_{q}^{-}(\omega)\right) \equiv S_{q}-S_{0}
$$

When this equation has a solution for $q$ with positive real part the corresponding steady state is unstable, and otherwise it is stable. 


\section{Appendix V.}

\section{Cracks which go faster when pulled harder are stable}

This Appendix derives relation Eq. (4.39).

Notice from Eq. (IV.4a) that

$$
z_{q}(a \omega, a v, b / a, q)=z_{q}(\omega, v, b, q-i a \omega+i \omega)
$$

Therefore

$$
Q_{q}(a \omega, a v, b / a, q)=Q_{q}(\omega, v, b, q-i a \omega+i \omega)
$$

(since the additional dependence of $Q_{q}$ upon $\omega$ and $v$ is of the form $\omega / v$ )

$$
\Rightarrow \frac{\partial Q_{q}}{\partial q}=i \frac{\partial Q_{q}}{\partial \omega}-\frac{v}{i \omega} \frac{\partial Q_{q}}{\partial v}+\frac{b}{i \omega} \frac{\partial Q_{q}}{\partial b} .
$$

Now

$$
Q_{q}^{-}(\omega)=\exp \left[\int_{-\infty}^{0} d t e^{i \omega t} \int \frac{d \omega^{\prime}}{2 \pi} e^{-i \omega^{\prime} t} \ln Q_{q}\left(\omega^{\prime}\right)\right]
$$

so

$$
\frac{\partial Q_{q}^{-}}{\partial q}=Q_{q}^{-} \int_{-\infty}^{0} d t e^{i \omega t} \int \frac{d \omega^{\prime}}{2 \pi} e^{-i \omega^{\prime} t} \frac{\partial}{\partial q} \ln Q_{q}\left(\omega^{\prime}\right),
$$

which implies, since $Q_{q}$ depends upon $q$ only through $z$, that

$$
\frac{\partial Q_{q}^{-}}{\partial q}=Q_{q}^{-} \int_{-\infty}^{0} d t e^{i \omega t} \int \frac{d \omega^{\prime}}{2 \pi} e^{-i \omega^{\prime} t}\left\{i \frac{\partial}{\partial \omega^{\prime}}-\frac{v}{i \omega^{\prime}} \frac{\partial}{\partial v}+\frac{b}{i \omega \prime} \frac{\partial}{\partial b}\right\} \ln Q_{q}\left(\omega^{\prime}\right) .
$$

Considering the terms in Eq. (V.5) one by one, we have first

$$
\begin{gathered}
\int_{-\infty}^{0} d t e^{i \omega t} \int \frac{d \omega^{\prime}}{2 \pi} e^{-i \omega^{\prime} t} i \frac{\partial}{\partial \omega^{\prime}} \ln Q_{q} \\
=\int_{-\infty}^{0} d t e^{i \omega t} \int \frac{d \omega^{\prime}}{2 \pi} e^{-i \omega^{\prime} t} i(i t) \ln Q_{q} \\
=i \frac{\partial}{\partial \omega} \int_{-\infty}^{0} d t e^{i \omega t} \int \frac{d \omega^{\prime}}{2 \pi} e^{-i \omega^{\prime} t} \ln Q_{q} \\
=i \frac{\partial}{\partial \omega} \ln Q_{q}^{-}(\omega)
\end{gathered}
$$

Second,

$$
\begin{aligned}
& \int_{-\infty}^{0} d t e^{i \omega t} \int \frac{d \omega^{\prime}}{2 \pi} e^{-i \omega^{\prime} t} \frac{1}{i \omega^{\prime}} \ln Q_{q} \\
& =\lim _{\epsilon \rightarrow 0} \int \frac{d \omega}{2 \pi} \frac{e^{-i \epsilon \omega^{\prime}}}{i\left(\omega-\omega^{\prime}\right)} \frac{1}{i \omega^{\prime}} \ln Q_{q}^{-}
\end{aligned}
$$




$$
\begin{gathered}
=\lim _{\epsilon \rightarrow 0} \int \frac{d \omega}{2 \pi} \frac{e^{-i \epsilon \omega^{\prime}}}{i \omega}\left[\frac{1}{i\left(\omega-\omega^{\prime}\right)}+\frac{1}{i \omega^{\prime}}\right] \ln Q_{q}^{-} \\
=\frac{1}{i \omega} \ln \left(\frac{Q_{q}^{-}(\omega)}{Q_{q}^{-}(0)}\right)
\end{gathered}
$$

Putting together Eq. (V.6) and Eq. (V.7) gives that

$$
\frac{\partial}{\partial q} \ln Q_{q}^{-}(\omega)=\left[i \frac{\partial}{\partial \omega}-\frac{v}{i \omega} \frac{\partial}{\partial v}+\frac{b}{i \omega} \frac{\partial}{\partial b}\right] \ln \left(\frac{Q_{q}^{-}(\omega)}{Q_{q}^{-}(0)}\right) .
$$

Returning with this result to Eq. (4.37) gives

$$
S_{0}^{\prime}=\int \frac{d \omega}{2 \pi} Q_{0}^{-}(\omega) \frac{v}{i \omega} \frac{\partial}{\partial v} \ln \left(\frac{Q_{0}^{-}(\omega)}{Q_{0}^{-}(0)}\right) .
$$

Since

$$
Q_{q}^{-}(\infty)=1
$$

one has finally that

$$
\begin{gathered}
S_{0}^{\prime}=-v \frac{\partial}{\partial v} \ln Q_{0}^{-}(0)=v \frac{\partial}{\partial v} \ln \frac{u(0)}{\Delta} \\
\Rightarrow S_{0}^{\prime}=-\frac{\partial \ln \Delta}{\partial \ln v}
\end{gathered}
$$

An additional expression makes it possible to evaluate $S_{q}$ more quickly, and without needing recourse to Fourier transforms. One has that

$$
\begin{gathered}
\int \frac{d \omega}{2 \pi}\left[Q_{q}^{-}(\omega)-1\right]=\lim _{\omega \rightarrow \infty} i \omega\left[Q_{q}^{-}(\omega)-1\right] . \\
=\lim _{\omega \rightarrow \infty} i \omega\left\{\exp \left[\int_{-\infty}^{0} d t e^{i \omega t} \int \frac{d \omega^{\prime}}{2 \pi} e^{-i \omega^{\prime} t} \ln Q_{q}\left(\omega^{\prime}\right)\right]-1\right\} .
\end{gathered}
$$

The large $\omega$ behavior in the exponential is given by the discontinuity in the function of $t$ at 0 , so one has next

$$
\begin{gathered}
\lim _{\omega \rightarrow \infty} i \omega\left\{\exp \left[\frac{1}{i \omega} \int \frac{d \omega^{\prime}}{2 \pi} \ln Q_{q}\left(\omega^{\prime}\right)\right]-1\right\} \\
=\int \frac{d \omega^{\prime}}{2 \pi} \ln Q_{q}\left(\omega^{\prime}\right) .
\end{gathered}
$$

One has finally that

$$
S_{q}=\int \frac{d \omega^{\prime}}{2 \pi} \ln \left[\frac{Q_{0}\left(\omega^{\prime}\right)}{Q_{q}\left(\omega^{\prime}\right)}\right]
$$




\section{Appendix VI.}

\section{Calculation of $Q$ for fully two-dimensional model}

This Appendix describes the calculation of $Q$ for the model of Section 5. There seems no simple way to report it, and the calculations were all carried out in MAXIMA; the batch file is available upon request from the first author.

Let $\Delta_{i}$ describe the displacements between points as shown in Fig. 5.1. The restoring force parallel to the direction of equilibrium bonds will be $k_{\|}$, while that perpendicular to this direction will be $k_{\perp}$. The force due to the displacement of the particle along $\vec{\Delta}_{1}=\vec{u}_{i-1, j+1}-\vec{u}_{i, j}$ is

$$
\begin{gathered}
k_{\|} \hat{d}_{\| 1}\left(\vec{\Delta}_{1} \cdot \hat{d}_{\| 1}\right)+k_{\perp} \hat{d}_{\perp 1}\left(\vec{\Delta}_{1} \cdot \hat{d}_{\perp 1}\right) \\
=k_{\|}\left(\frac{-1}{2}, \frac{\sqrt{3}}{2}\right)\left(\frac{-1}{2} \Delta_{1}^{x}, \frac{\sqrt{3}}{2} \Delta_{1}^{y}\right)+k_{\perp}\left(\frac{\sqrt{3}}{2}, \frac{1}{2}\right)\left(\frac{\sqrt{3}}{2} \Delta_{1}^{x}, \frac{1}{2} \Delta_{1}^{y}\right)
\end{gathered}
$$

Adding up contributions from other particles in this way we get for the force due to neighbors

$$
\vec{F}(m, n)=\sum_{j=1}^{6} \sum_{q=\|, \perp} k_{q} \hat{d}_{q i}\left(\vec{\Delta}_{i}(m, n) \cdot \hat{d}_{q i}\right)
$$

In steady state, for $n>1 / 2$ the forces become

$$
\begin{gathered}
\left(\frac{1}{4} k_{\|}+\frac{3}{4} k_{\perp}\right)\left[\begin{array}{c}
u_{n+1}^{x}\left(t-\left(g_{n+1}-1\right) a / v\right)+u_{n+1}^{x}\left(t-g_{n+1} a / v\right) \\
+u_{n-1}^{x}\left(t-\left(g_{n+1}-1\right) a / v\right)+u_{n-1}^{x}\left(t-g_{n+1} a / v\right)
\end{array}-4 u_{n}^{x}(t)\right] \\
F^{x}=+\frac{\sqrt{3}}{4}\left(k_{\perp}-k_{\|}\right)\left[\begin{array}{c}
u_{n+1}^{y}\left(t-\left(g_{n+1}-1\right) a / v\right)-u_{n+1}^{y}\left(t-g_{n+1} a / v\right) \\
-u_{n-1}^{y}\left(t-\left(g_{n+1}-1\right) a / v\right)+u_{n-1}^{y}\left(t-g_{n+1} a / v\right)
\end{array}\right] \\
+k_{\|}\left[u_{n}^{x}(t+a / v)+u_{n}^{x}(t-a / v)-2 u_{n}^{x}(t)\right]
\end{gathered}
$$

In Eq. (VI.4), all the displacements $u$ are evaluated at $m=0$, and this index is therefore dropped, so that the only remaining index refers to the layer number $n$. For $n=1 / 2$, one 
has instead

$$
\begin{aligned}
& \left(\frac{1}{4} k_{\|}+\frac{3}{4} k_{\perp}\right)\left[\begin{array}{l}
u_{n+1}^{x}\left(t-\left(g_{n+1}-1\right) a / v\right)+u_{n+1}^{x}\left(t-g_{n+1} a / v\right) \\
+\theta(-t)\left(u_{n-1}^{x}\left(t-\left(g_{n+1}-1\right) a / v\right)-u_{n}^{x}(t)\right) \\
+\theta(a / 2 v-t)\left(u_{n-1}^{x}\left(t-g_{n+1} a / v\right)-u_{n}^{x}(t)\right)
\end{array}\right] \\
& F^{x}=+\frac{\sqrt{3}}{4}\left(k_{\perp}-k_{\|}\right)\left[\begin{array}{c}
u_{n+1}^{y}\left(t-\left(g_{n+1}-1\right) a / v\right)-u_{n+1}^{y}\left(t-g_{n+1} a / v\right) \\
-\theta(-t)\left(u_{n-1}^{y}\left(t-\left(g_{n+1}-1\right) a / v\right)-u_{n}^{y}(t)\right) \\
+\theta(a / 2 v-t)\left(u_{n-1}^{y}\left(t-g_{n+1} a / v\right)-u_{n}^{y}(t)\right)
\end{array}\right] \\
& +k_{\|}\left[u_{n}^{x}(t+a / v)+u_{n}^{x}(t-a / v)-2 u_{n}^{x}(t)\right] \\
& \left(\frac{1}{4} k_{\perp}+\frac{3}{4} k_{\|}\right)\left[\begin{array}{ll}
u_{n+1}^{y}\left(t-\left(g_{n+1}-1\right) a / v\right)+u_{n+1}^{y}\left(t-g_{n+1} a / v\right) \\
+\theta(-t)\left(u_{n-1}^{y}\left(t-\left(g_{n+1}-1\right) a / v\right)-u_{n}^{y}(t)\right) & -2 u_{n}^{y}(t) \\
+\theta(a / 2 v-t)\left(u_{n-1}^{y}\left(t-g_{n+1} a / v\right)-u_{n}^{y}(t)\right)
\end{array}\right] \\
& F^{y}=+\frac{\sqrt{3}}{4}\left(k_{\perp}-k_{\|}\right)\left[\begin{array}{c}
u_{n+1}^{x}\left(t-\left(g_{n+1}-1\right) a / v\right)-u_{n+1}^{x}\left(t-g_{n+1} a / v\right) \\
-\theta(t)\left(u_{n-1}^{x}\left(t-\left(g_{n+1}-1\right) a / v\right)-u_{n}^{x}(t)\right) \\
+\theta(a / 2 v-t)\left(u_{n-1}^{x}\left(t-g_{n+1} a / v\right)-u_{n}^{x}(t)\right)
\end{array}\right] \\
& +k_{\perp}\left[u_{n}^{y}(t+a / v)+u_{n}^{y}(t-a / v)-2 u_{n}^{y}(t)\right]
\end{aligned}
$$

Now take the Fourier transform in time of these equations. For the layers with $n>1 / 2$ one has

$$
\begin{aligned}
& \left(\frac{1}{4} k_{\|}+\frac{3}{4} k_{\perp}\right)\left[\begin{array}{c}
u_{n+1}^{x}(\omega)\left(e^{i \omega\left(g_{n+1}-1\right) a / v}+e^{i \omega g_{n+1} a / v}\right) \\
+u_{n-1}^{x}(\omega)\left(e^{i \omega\left(g_{n+1}-1\right) a / v}+e^{\left(t-g_{n+1} a / v\right)}\right)
\end{array}-4 u_{n}^{x}(\omega)\right] \\
& F^{x}=+\frac{\sqrt{3}}{4}\left(k_{\perp}-k_{\|}\right)\left[\begin{array}{r}
u_{n+1}^{y}(\omega)\left(e^{i \omega\left(g_{n+1}-1\right) a / v}-e^{i \omega g_{n+1} a / v}\right) \\
-u_{n-1}^{y}(\omega)\left(e^{i \omega\left(g_{n+1}-1\right) a / v}-e^{i \omega g_{n+1} a / v}\right)
\end{array}\right] \\
& +k_{\|}\left[u_{n}^{x}(\omega) e^{i \omega a / v}+e^{-i \omega a / v}-2\right] \\
& \left(\frac{1}{4} k_{\perp}+\frac{3}{4} k_{\|}\right)\left[\begin{array}{c}
u_{n+1}^{y}(\omega)\left(e^{i \omega\left(g_{n+1}-1\right) a / v}+e^{i \omega g_{n+1} a / v}\right) \\
+u_{n-1}^{y}(\omega)\left(e^{i \omega\left(g_{n+1}-1\right) a / v}+e^{\left(t-g_{n+1} a / v\right)}\right)
\end{array}-4 u_{n}^{y}(\omega)\right] \\
& F^{y}=+\frac{\sqrt{3}}{4}\left(k_{\perp}-k_{\|}\right)\left[\begin{array}{r}
u_{n+1}^{x}(\omega)\left(e^{i \omega\left(g_{n+1}-1\right) a / v}-e^{i \omega g_{n+1} a / v}\right) \\
-u_{n-1}^{x}(\omega)\left(e^{i \omega\left(g_{n+1}-1\right) a / v}-e^{i \omega g_{n+1} a / v}\right)
\end{array}\right] \\
& +k_{\perp}\left[u_{n}^{y}(\omega) e^{i \omega a / v}+e^{-i \omega a / v}-2\right]
\end{aligned}
$$


Substituting in the form

$$
\left(\begin{array}{c}
u_{n}^{x} \\
u_{n}^{y}
\end{array}\right)=y^{n} e^{-i \omega g_{n} / 2 v}\left(\begin{array}{c}
U_{x} \\
U_{y}
\end{array}\right)
$$

gives

$$
\begin{gathered}
-\left(m \omega^{2}+i \omega b(\omega)\right) U_{x}=\left\{\begin{array}{r}
\left(k_{\|}+3 k_{\perp}\right) \cos (\omega a / 2 v) \frac{\left(y+y^{-1}\right)}{2} \\
+2 k_{\|} \cos (\omega a / v)-3\left(k_{\perp}+k_{\|}\right)
\end{array}\right\} U_{x} \\
-\sqrt{3} i\left(k_{\perp}-k_{\|}\right) \sin (\omega a / 2 v) \frac{\left(y-y^{-1}\right)}{2} U_{y} \\
-\left(m \omega^{2}+i \omega b(\omega)\right) U_{y}=\left\{\begin{array}{c}
\left(k_{\perp}+3 k_{\|}\right) \cos (\omega a / 2 v) \frac{\left(y+y^{-1}\right)}{2} \\
+2 k_{\perp} \cos (\omega a / v)-3\left(k_{\perp}+k_{\|}\right) \\
-\sqrt{3} i\left(k_{\perp}-k_{\|}\right) \sin (\omega a / 2 v) \frac{\left(y-y^{-1}\right)}{2} U_{x} .
\end{array}\right.
\end{gathered}
$$

Here, and in what follows, the dissipation coefficient $b$ is understood to be of the form

$$
b(\omega)=b_{0} \sqrt{\omega^{2}+\omega_{0}^{2}}
$$

The condition that the determinant of this system vanish determines $y$ by

$$
\begin{aligned}
& {\left[\begin{array}{c}
\left(k_{\|}+3 k_{\perp}\right) \cos (\omega a / 2 v) \frac{\left(y+y^{-1}\right)}{2} \\
+m \omega^{2}+i \omega b+2 k_{\|} \cos (\omega a / v)-3\left(k_{\perp}+k_{\|}\right)
\end{array}\right]\left[\begin{array}{c}
\left(k_{\perp}+3 k_{\|}\right) \cos (\omega a / 2 v) \frac{\left(y+y^{-1}\right)}{2} \\
+m \omega^{2}+i \omega b+2 k_{\perp} \cos (\omega a / v)-3\left(k_{\perp}+k_{\|}\right)
\end{array}\right] . } \\
& +3\left[\left(k_{\perp}-k_{\|}\right) \sin (\omega a / 2 v) \frac{\left(y-y^{-1}\right)}{2}\right]^{2}
\end{aligned}
$$

It is more convenient to define

$$
z=\frac{y+1 / y}{2} .
$$

Then the determinental condition becomes

$$
\begin{aligned}
& A=3\left(k_{\perp}-k_{\|}\right)^{2}+16 k_{\perp} k_{\|} \cos (a \omega /(2 v))^{2} \\
& B=\cos (a \omega / 2 v)\left(2\left(3 k_{\|}^{2}+2 k_{\|} k_{\perp}+3 k_{\perp}^{2}\right) \cos (a \omega / v)+4\left(k_{\perp}+k_{\|}\right)\left(m \omega^{2}+i \omega b-3\left(k_{\|}+k_{\perp}\right)\right)\right) \\
& C=\left(m \omega^{2}+i \omega b-\left(k_{\|}+k_{\perp}\right)(3-\cos (a \omega / v))\right)^{2}-\left(k_{\|}-k_{\perp}\right)^{2}\left(\cos (a \omega / v)^{2}+3 \sin (a \omega /(2 v))^{2}\right) . \\
& z_{ \pm}=\frac{-B \pm \sqrt{B^{2}-4 A C}}{2 A}
\end{aligned}
$$


There are now 4 values of $y$ which satisfy Eq. (VI.10) for any given $\omega$, namely

$$
y_{ \pm}=z_{ \pm}+\sqrt{\left(z_{ \pm}\right)^{2}-1}
$$

and two others given by the inverse of these, or equivalently by changing the sign of the square root.

Define

$$
\begin{aligned}
D_{ \pm}= & m \omega^{2}+i \omega b+\left(k_{\|}+3 k_{\perp}\right) \cos (\omega a / 2 v) \frac{\left(y_{ \pm}+y_{ \pm}^{-1}\right)}{2} \\
& +2 k_{\|} \cos (\omega a / v)-3\left(k_{\perp}+k_{\|}\right) \\
E_{ \pm}= & -\sqrt{3} i\left(k_{\perp}-k_{\|}\right) \sin (\omega a / 2 v) \frac{\left(y^{p} m-y_{ \pm}^{-1}\right)}{2}
\end{aligned}
$$

Then a general solution of Eq. (VI.6) is

$$
\begin{aligned}
& \left(\begin{array}{c}
u_{n}^{x} \\
u_{n}^{y}
\end{array}\right)=e^{-i \omega g_{n} / 2 v}\left[\begin{array}{cc}
y_{+}^{(n-1 / 2)}\left(\begin{array}{c}
E_{+} \\
-D_{+}
\end{array}\right) u_{1+}+y_{+}^{(-n+1 / 2)}\left(\begin{array}{c}
E_{+} \\
D_{+}
\end{array}\right) u_{2+} \\
+y_{-}^{(n-1 / 2)}\left(\begin{array}{c}
E_{-} \\
-D_{-}
\end{array}\right) u_{1-}+y_{-}^{(-n+1 / 2)}\left(\begin{array}{c}
E_{-} \\
D_{-}
\end{array}\right) u_{2-}
\end{array}\right] . \\
& +U_{N} \frac{(n-1 / 2)}{N}\left(\begin{array}{l}
0 \\
1
\end{array}\right)
\end{aligned}
$$

The four functions $u_{1 \pm}, u_{2 \pm}$ can be determined from the four conditions

$$
u_{N+1 / 2}^{x}=u_{N+1 / 2}^{y}-U_{N}=0
$$

and

$$
\left(\begin{array}{c}
u_{1 / 2}^{x} \\
u_{1 / 2}^{y}
\end{array}\right)=\left[\left(\begin{array}{c}
E_{+} \\
-D_{+}
\end{array}\right) u_{1+}+\left(\begin{array}{c}
E_{+} \\
D_{+}
\end{array}\right) u_{2+}+\left(\begin{array}{c}
E_{-} \\
-D_{-}
\end{array}\right) u_{1-}+\left(\begin{array}{c}
E_{-} \\
D_{-}
\end{array}\right) u_{2-}\right]
$$

Once they are determined, one can obtain in particular a solution for $u_{3 / 2}$, in terms of $u_{1 / 2}^{x}$ and $u_{1 / 2}^{y}$; however, the expressions are too long to list here explicitly.

Thus the problem is reduced to that of finding $u_{1 / 2}^{x}$ and $u_{1 / 2}^{y}$. These are determined by taking the Fourier transform of the equations on the line $n=1 / 2$. Unfortunately, we do not know how to solve the equations in full generality. The one restriction that must be imposed is that right on the crack line, we must take $k_{\perp}=0$. Otherwise the formalism dies. We would like very much to overcome this restriction, but do not now see how to do it. However, to try to make up for it, we will let $k_{\|}$equal some arbitrary $k_{\|}^{I}$ on the 
interface. Given this restriction, Eq. (VI.6) becomes

$$
\begin{aligned}
& \left(\frac{1}{4} k_{\|}+\frac{3}{4} k_{\perp}\right)\left[u_{3 / 2}^{x}(t)+u_{3 / 2}^{x}(t-a / v)-2 u_{1 / 2}^{x}(t)\right] \\
& +\frac{k_{\|}^{I}}{4} \theta(-t)\left(u_{-1 / 2}^{x}(t)-u_{1 / 2}^{x}(t)\right) \\
& {\left[+\frac{k_{\|}^{I}}{4} \theta(a / 2 v-t)\left(u_{-1 / 2}^{x}(t-a / v)-u_{1}^{x} / 2(t)\right)\right.} \\
& F^{x}=+\frac{\sqrt{3}}{4}\left[\begin{array}{c}
\left(k_{\perp}-k_{\|}\right)\left[u_{3 / 2}^{y}(t)-u_{3 / 2}^{y}(t-a / v)\right] \\
+k_{\|}^{I} \theta(-t)\left(u_{-1 / 2}^{y}(t)-u_{1 / 2}^{y}(t)\right) \\
-k_{\|}^{I} \theta(a / 2 v-t)\left(u_{-1 / 2}^{y}(t-a / v)-u_{1 / 2}^{y}(t)\right)
\end{array}\right] \\
& +k_{\|}\left[u_{1 / 2}^{x}(t+a / v)+u_{1 / 2}^{x}(t-a / v)-2 u_{1 / 2}^{x}(t)\right] \\
& F^{y}=\left[\begin{array}{c}
\left(\frac{1}{4} k_{\perp}+\frac{3}{4} k_{\|}\right)\left[u_{3 / 2}^{y}(t)+u_{3 / 2}^{y}(t-a / v)-2 u_{1 / 2}^{y}(t)\right] \\
+\frac{3 k_{\|}^{I}}{4} \theta(-t)\left(u_{-1 / 2}^{y}(t)-u_{1 / 2}^{y}(t)\right) \\
+\frac{3 k_{\|}^{I}}{4} \theta(a / 2 v-t)\left(u_{-1 / 2}^{y}(t-a / v)-u_{1 / 2}^{y}(t)\right)
\end{array}\right] \\
& +\frac{\sqrt{3}}{4}\left[\begin{array}{c}
\left(k_{\perp}-k_{\|}\right)\left[u_{3 / 2}^{x}(t)-u_{3 / 2}^{x}(t-a / v)\right] \\
+k_{\|}^{I} \theta(-t)\left(u_{-1 / 2}^{x}(t)-u_{1 / 2}^{x}(t)\right) \\
-k_{\|} \theta(a / 2 v-t)\left(u_{-1 / 2}^{x}(t-a / v)-u_{1 / 2}^{x}(t)\right)
\end{array}\right] \\
& +k_{\perp}\left[u_{1 / 2}^{y}(t+a / v)+u_{1 / 2}^{y}(t-a / v)-2 u_{1 / 2}^{y}(t)\right]
\end{aligned}
$$

The important property of this set of equations is that there is really only one linear combination of $u^{x}$ and $u^{y}$ which multiplies the $\theta$ functions. This combination is

$$
U(t)=\frac{-1}{2 \sqrt{3}}\left[u_{1 / 2}^{x}(t+a / 2 v)-u_{1 / 2}^{x}(t)\right]+\frac{1}{2}\left\{u_{1 / 2}^{y}(t+a / 2 v)+u_{1 / 2}^{y}(t)\right\}
$$

To see why it enters, notice that when the strip is loaded in Mode I, one must have the symmetries

$$
\begin{aligned}
& u_{-1 / 2}^{y}(t)=-u_{1 / 2}^{y}(t+a / 2 v) \\
& u_{-1 / 2}^{x}(t)=u_{1 / 2}^{x}(t+a / 2 v) .
\end{aligned}
$$


This symmetry allows one to eliminate the fields with subscript $-1 / 2$ from Eq. (VI.18). The result is

$$
\begin{aligned}
& \left(\frac{1}{4} k_{\|}+\frac{3}{4} k_{\perp}\right)\left[u_{3 / 2}^{x}(t)+u_{3 / 2}^{x}(t-a / v)-2 u_{1 / 2}^{x}(t)\right] \\
& F^{x}=\begin{array}{c}
+\frac{\sqrt{3}}{4}\left(k_{\perp}-k_{\|}\right)\left[u_{3 / 2}^{y}(t)-u_{3 / 2}^{y}(t-a / v)\right] \\
+k_{\|}\left[u_{1 / 2}^{x}(t+a / v)+u_{1 / 2}^{x}(t-a / v)-2 u_{1 / 2}^{x}(t)\right]
\end{array} \\
& -\frac{k_{\|}^{I}}{8 \sqrt{3}}[U(t) \theta(-t)-U(t-a / 2 v) \theta(a / 2 v-t)] \\
& \left(\frac{1}{4} k_{\perp}+\frac{3}{4} k_{\|}\right)\left[u_{3 / 2}^{y}(t)+u_{3 / 2}^{y}(t-a / v)-2 u_{1 / 2}^{y}(t)\right] \\
& \begin{aligned}
F^{y}= & +\frac{\sqrt{3}}{4}\left(k_{\perp}-k_{\|}\right)\left[u_{3 / 2}^{x}(t)-u_{3 / 2}^{x}(t-a / v)\right] \\
+ & k_{\perp}\left[u_{1 / 2}^{y}(t+a / v)+u_{1 / 2}^{y}(t-a / v)-2 u_{1 / 2}^{y}(t)\right]
\end{aligned} \\
& -\frac{k_{\|}^{I}}{8}[U(t) \theta(-t)+U(t-a / 2 v) \theta(a / 2 v-t)]
\end{aligned}
$$

It is now possible to Fourier transform Eq. (VI.21). The result is

$$
\begin{aligned}
& \left(\frac{1}{4} k_{\|}+\frac{3}{4} k_{\perp}\right)\left[u_{3 / 2}^{x}(\omega)\left(1+e^{i \omega a / v}\right)-2 u_{1 / 2}^{x}(\omega)\right] \\
& -\left(m \omega^{2}+i b \omega\right) u_{1 / 2}^{x}(\omega)=\begin{array}{l}
+\frac{\sqrt{3}}{4}\left(k_{\perp}-k_{\|}\right)\left[u_{3 / 2}^{y}(\omega)\left(1-e^{i \omega a / v}\right)\right] \\
+k_{\|}\left[2 u_{1 / 2}^{x} \cos (a \omega / v)-2 u_{1 / 2}^{x}(\omega)\right]
\end{array} \\
& -\frac{k_{\|}^{I}}{8 \sqrt{3}} U^{-}(\omega)\left(1-e^{i \omega a / 2 v}\right) \\
& \left(\frac{1}{4} k_{\perp}+\frac{3}{4} k_{\|}\right)\left[u_{3 / 2}^{y}(\omega)\left(1+e^{i \omega a / v}\right)-2 u_{1 / 2}^{y}(\omega)\right]
\end{aligned}
$$

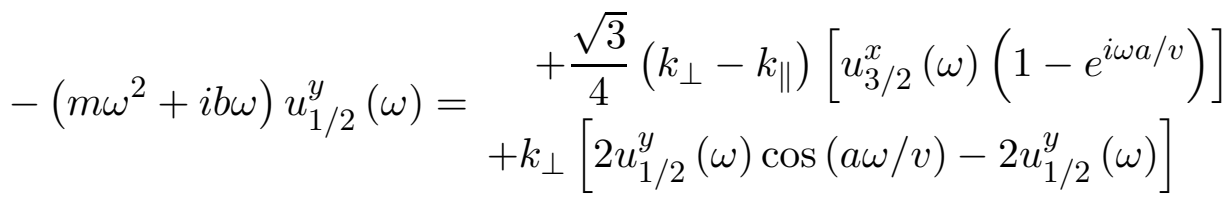

$$
\begin{aligned}
& -\frac{k_{\|}^{I}}{8} U^{-}(\omega)\left(1+e^{i \omega a / v}\right)
\end{aligned}
$$

Using Eq. (VI.15) to find $u_{3 / 2}^{x}$ and $u_{3 / 2}^{y}$, and Eq. (VI.19) to find $U(\omega)$, one can eliminate all variables but $U$ from Eq. (VI.22). 
Analyzing the $\omega \rightarrow 0$ behavior, where

$$
\begin{aligned}
& u_{1 / 2}^{x} \rightarrow 0 \\
& U \rightarrow u_{1 / 2}^{y}, \\
& u_{3 / 2}^{y} \rightarrow(1-1 / N) u_{1 / 2}^{y}+U_{N} / N \delta(\omega)
\end{aligned}
$$

one finds finally that

$$
Q(\omega) U^{+}+U^{-}=Q_{0} U_{N} \delta(\omega)
$$

as in Section 3. The expression for $Q$ is again too lengthy to record here. 